Journal of Computational Neuroscience, 1, 195-230 (1994)

(c) 1994 Kluwer Academic Publishers. Manufactured in The Netherlands.

\title{
Synthesis of Models for Excitable Membranes, Synaptic Transmission and Neuromodulation Using a Common Kinetic Formalism
}

\author{
ALAIN DESTEXHE* AND ZACHARY F. MAINEN \\ The Howard Hughes Medical Institute and The Salk Institute for Biological Studies, Computational \\ Neurobiology Laboratory, 10010 North Torrey Pines Road, La Jolla, CA 92037, USA \\ TERRENCE J. SEJNOWSKI \\ The Howard Hughes Medical Institute and The Salk Institute for Biological Studies, Computational \\ Neurobiology Laboratory, 10010 North Torrey Pines Road, La Jolla, CA 92037, USA and \\ Dept. of Biology, University of California-San Diego, La Jolla, CA 92037
}

Received January 14, 1994; Revised March 24, 1994; Accepted (in revised form) April 12, 1994

Action Editor: L. Abbott

\begin{abstract}
Markov kinetic models were used to synthesize a complete description of synaptic transmission, including opening of voltage-dependent channels in the presynaptic terminal, release of neurotransmitter, gating of postsynaptic receptors, and activation of second-messenger systems. These kinetic schemes provide a more general framework for modeling ion channels than the Hodgkin-Huxley formalism, supporting a continuous spectrum of descriptions ranging from the very simple and computationally efficient to the highly complex and biophysically precise. Examples are given of simple kinetic schemes based on fits to experimental data that capture the essential properties of voltage-gated, synaptic and neuromodulatory currents. The Markov formalism allows the dynamics of ionic currents to be considered naturally in the larger context of biochemical signal transduction. This framework can facilitate the integration of a wide range of experimental data and promote consistent theoretical analysis of neural mechanisms from molecular interactions to network computations.
\end{abstract}

\section{Introduction}

More than forty years ago, Hodgkin and Huxley (1952) introduced an extremely influential description of voltage-dependent ionic currents. Their model accurately described the macroscopic currents underlying action potentials in the squid giant axon and postulated an explanation for the voltage-sensitivity of the currents based on independent gating particles. The Hodgkin-Huxley paradigm has been extended subsequently to describe a large range of other voltage-dependent channels, and is widely used today in most neuronal models that use ion channels.

The biophysical properties of single channels have been studied in depth using patch record-

\footnotetext{
${ }^{*}$ To whom correspondence should be addressed.
}

ing techniques (Sakmann and Neher, 1983). Singlechannel recordings have shown that the channels undergo rapid transitions between conducting and nonconducting states. It is now known that conformational changes of the channel protein, rather than movement of gating particles per se, give rise to the voltage-sensitivity of ion currents. Conformational changes can be described by a state diagram that is analogous to the equations used to describe chemical reactions.

Markov models are a class of kinetic scheme based on state diagrams in which the probabilities of state transitions are time-independent. These models have been used for well over a decade to model the gating characteristics of many voltage-dependent ion channels and ligand-gated receptors. In particular, Markov models can account for many details of single-channel data, including open time distribu- 
tions and bursts of openings (Colquhoun and Hawkes, 1981). When reevaluated in light of single channel data, the Hodgkin-Huxley description has remained accurate in some respects but in many detailed ways has been superseded (Armstrong, 1992). The vast majority of biophysical studies now make use of Markov models to describe ionic conductances.

Synaptic currents in computational models are usually described using alpha functions or related double-exponential waveforms fit to recordings of postsynaptic currents (PSCs). These waveforms are convenient and computationally efficient, but have several deficiencies. First, alpha functions can approximate only a limited class of synaptic potentials, and correspond to a simple first-order linear biophysical scheme (see Appendix B): for central synapses, the rise time is usually much faster than the decay time constant whereas there is only a single time constant for an alpha function. Second, alpha functions do not provide naturally for the summation or saturation of postsynaptic currents, which may be critical for models involving high-frequency bursting neurons (see Destexhe et al., 1993b, 1994a). Finally, the use of a fixed conductance template such as the alpha function complicates the analysis of the neuron as a dynamical system.

The first quantitative descriptions of currents at the neuromuscular junction used kinetic equations of a simple Markovian form (Katz, 1966; Magleby and Stevens, 1972). Subsequent application of singlechannel recordings to the study of synaptic transmission (Neher, 1992; Sakmann, 1992) has provided many increasingly detailed models which continue to use Markov kinetic schemes, yet alpha functions remain the favored description for PSCs among computational modelers (Koch and Segev, 1989).

Although the Hodgkin-Huxley formalism for voltage-dependent channels and alpha functions for synaptic currents have been useful for many purposes, there are many advantages to replacing both with a uniform approach based on Markov kinetics. First, years of biophysical studies have shown that Markov models provide descriptions sufficiently general and powerful to capture the behavior of channels even when revealed directly through single-channel recordings. Second, kinetic models in general are comprehensive enough to describe not only ion channels but the entire array of enzymes and molecular interactions that underlie signalling in nerve cells. Finally, the state diagrams of Markov models can be related directly to the structure of the underlying molecules and therefore adapted as our molecular understanding progresses.

In this paper, we show that the kinetic approach is a comprehensive tool for biophysical and molecular studies as well as a flexible enough framework to produce useful computational neural models. We synthesize a model of synaptic transmission from invasion of the presynaptic terminal by action potentials, through calcium-dependent release, to action of both fast and slow (modulatory) postsynaptic currents. Both detailed and simplified Markov models are examined, and simulated synaptic currents are compared to whole-cell voltage-clamp recordings. The essential properties of voltage-dependent channels, transmittergated channels and second messenger-activated channels are well-captured by even relatively simple kinetic schemes that are computationally efficient and amenable to analysis.

\section{Methods}

All ionic currents considered in this paper were given by models of ion channels described by a kinetic formalism for the transitions between their conformational states. All simulations were performed with the NEURON compartmental simulation program (Hines, 1989, 1993). The kinetic equations were written and solved directly using KINETIC methods of the NMODL language of NEURON, which is a derivative of the MODL description language of the SCoP package (Kohn, 1989). In some cases, analytic expressions were obtained and compared with the simulations.

\subsection{Kinetic Description of Ion Channel Gating}

The description of gating kinetics at the singlechannel level makes use of state diagrams that have the same significance as the reaction diagrams in chemical kinetics

$$
S_{1} \rightleftarrows S_{2} \rightleftarrows \ldots \rightleftharpoons S_{n} \text {. }
$$

The various states, $S_{1} \ldots S_{n}$, are meant to represent a sequence of protein conformations that underlies the gating of a channel. Define $P\left(S_{i}, t\right)$ as the probability of being in a state $S_{i}$ at time $t$ and $P\left(S_{i} \rightarrow S_{j}\right)$ as 
the transition probability from state $S_{i}$ to state $S_{j}$. The time evolution of the probability of state $S_{i}$ is then described by the Master equation (Colquhoun and Hawkes, 1977):

$$
\begin{aligned}
\frac{d P\left(S_{i}, t\right)}{d t}= & \sum_{j=1}^{n} P\left(S_{j}, t\right) P\left(S_{j} \rightarrow S_{i}\right) \\
& -\sum_{j=1}^{n} P\left(S_{i}, t\right) P\left(S_{i} \rightarrow S_{j}\right) .
\end{aligned}
$$

The left term represents the "source" contribution of all transitions entering state $S_{i}$, and the right term represents the "sink" contribution of all transition leaving state $S_{i}$. In this equation, the time evolution depends only on the present state of the system, and is defined entirely by knowledge of the set of transition probabilities. Such systems are called Markovian systems.

In the case of a large number of identical channels or other proteins, the quantities given in the master equation can be reinterpreted. The probability of being in a state $S_{i}$ becomes the fraction of channels in state $S_{i}$, noted $s_{i}$, and the transition probabilities from state $S_{i}$ to state $S_{j}$ becomes the rate constants, $r_{i j}$, of the reactions

$$
S_{i} \underset{r_{j i}}{\stackrel{r_{i j}}{\rightleftarrows}} S_{j} .
$$

In this case, one can rewrite the master equation as:

$$
\frac{d s_{i}}{d t}=\sum_{j=1}^{n} s_{j} r_{j i}-\sum_{j=1}^{n} s_{i} r_{i j}
$$

which is a conventional kinetic equation for the various states of the system. We will refer to this type of system as Markov kinetics.

\subsection{Voltage-Dependent Channel Gating}

Voltage-dependent ion channels can be described using Markov kinetic schemes in which transition rates between some pairs of states, $i$ and $j$, vary as a function of the membrane potential, $V$ :

$$
S_{i} \underset{r_{j i}(V)}{\stackrel{r_{i j}(V)}{\rightleftarrows}} S_{j}
$$

Because the physical basis of the gating process is not well understood, a variety of forms for the voltage dependence are possible. According the theory of reaction rates (Johnson, Eyring and Stover, 1974), the rate of transition between two states depends exponentially on the free energy barrier between them. Thus

$$
r_{i j}(V)=\exp -U_{i j}(V) / R T,
$$

where $R$ is the gas constant and $T$ is the absolute temperature. The function $U_{i j}(V)$ is in general very difficult to ascertain, and may involve both linear and nonlinear components arising from interactions between the channel protein and the membrane electrical field. This dependence can be expressed without assumptions about underlying molecular mechanisms by a Taylor series expansion of the form

$$
U(V)=c_{o}+c_{1} V+c_{2} V^{2}+\ldots
$$

giving a general transition rate function

$r(V)=\exp \left[-\left(c_{o}+c_{1} V+c_{2} V^{2}+\ldots\right) / R T\right]$,

(Stevens, 1978), where $c_{0}, c_{1}, c_{2}, \ldots$ are constants which are specific for each transition. The constant $c_{0}$ corresponds to energy differences that are independent of the applied field, the linear term $c_{1} V$ to the translation of isolated charges or the rotation of rigid dipoles, and the higher order terms to effects such as electronic polarization and pressure induced by $V$ (Andersen, 1992). In the "low field limit" (during relatively small applied voltages), the contribution of the higher order terms may be negligible (Stevens, 1978; Andersen, 1992). Thus, a simple, commonlyused voltage dependence takes the form

$$
r_{i j}(V)=a_{i j} \exp \left(-V / b_{i j}\right)
$$

where $a_{i j}$ and $b_{i j}$ are constants.

Hodgkin-Huxley Formalism. The most elementary Markov model for a voltage-gated channel is the first order scheme

$$
C \underset{r_{2}(V)}{\stackrel{r_{1}(V)}{\rightleftarrows}} O,
$$

with voltage-gated rates $r_{1}(V)$ and $r_{2}(V)$ between a single open or conducting state, $O$, and a single closed state $C$. 
In the method of Hodgkin and Huxley (1952), several gates must open in order to activate a channel. Each gate has two states with the first order kinetics of Eq. 10, and each behaves entirely independently the others.

Gates are divided into two types, usually several gates for activation, $m$, and a single gate for inactivation, $h$. All activation gates are assumed to be identical to one another, reducing the number of state transitions that must be calculated to two

$$
\begin{aligned}
& m \underset{\beta_{m}(V)}{\stackrel{\alpha_{m}(V)}{\rightleftarrows}} m^{*} \\
& h \underset{\beta_{h}(V)}{\stackrel{\alpha_{h}(V)}{\rightleftarrows}} h^{*} .
\end{aligned}
$$

The channel conductance is simply calculated as the product of the open fractions of each gate

$$
o=m^{M} h
$$

where $M$ is the number of identical $m$ gates.

The Hodgkin-Huxley formalism is a subclass of the more general Markov representation. An equivalent Markov model can be written for any HodgkinHuxley scheme, but the translation of a system with multiple independent particles into a single-particle description results in a combinatorial explosion of states. Thus, the Markov model corresponding to the Hodgkin-Huxley sodium channel is

$$
\begin{aligned}
& C_{3} \stackrel{3 \alpha_{m}}{\underset{\beta_{m}}{\rightleftarrows}} C_{2} \stackrel{2 \alpha_{m}}{\underset{2 \beta_{m}}{\rightleftharpoons}} C_{1} \stackrel{\alpha_{m}}{\underset{3 \beta_{m}}{\rightleftharpoons}} O
\end{aligned}
$$

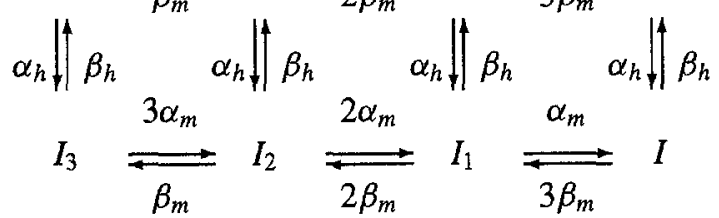

(FitzHugh, 1965). The states represent the channel with the inactivation gate in the open state (top) or closed state (bottom) and (from left to right) three, two, one or none of the activation gates closed. To reproduce the $m^{3}$ formulation, the rates must have the $3: 2: 1$ ratio in the forward direction and the $1: 2: 3$ ratio in the backward direction. Only the $O$ state is conducting. The squid delayed rectifier potassium current modeled by Hodgkin and Huxley (1952) with 4 activation gates and no inactivation can be treated analogously (Fitzhugh, 1965; Armstrong, 1965) giving

$$
C_{4} \stackrel{4 \alpha_{m}}{\underset{\beta_{m}}{\rightleftarrows}} C_{3} \underset{2 \beta_{m}}{\stackrel{3 \alpha_{m}}{\rightleftarrows}} C_{2} \underset{3 \beta_{m}}{\stackrel{2 \alpha_{m}}{\rightleftarrows}} C_{1} \underset{4 \beta_{m}}{\stackrel{\alpha_{m}}{\rightleftarrows}} O
$$

Markov Models of Voltage-Dependent Channels. In more general models using Markov kinetics, independent and identical gates are not assumed. Rather, a state diagram is written to represent the configuration of the entire channel protein. This relaxes the constraints on the form of the diagram and the ratios of rate constants imposed by the Hodgkin-Huxley formulation. One may begin with the elementary twostate scheme (Eq. 10) augmented by a single inactivated state, giving

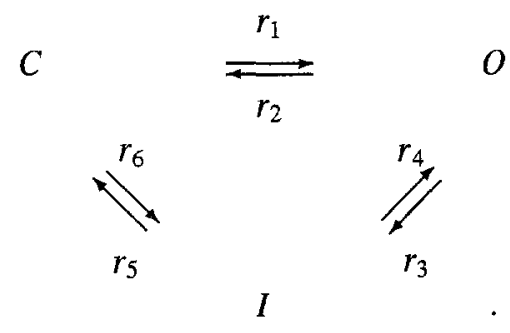

All six possible transitions between the three states are allowed, giving this kinetic scheme a looped form. The transition rates may follow voltage-dependent equations in the general form of Eq. (8) or some of these rates may be taken as either zero or independent of voltage to yield more simple models (see Results).

To fit more accurately the time course of channel openings or gating currents, additional closed and inactivated states may be necessary. As an example of a biophysically-derived multi-state Markov model, the squid sodium channel model of Vandenberg and Bezanilla (1991) was considered. The authors fit by least squares a combination of single channel, macroscopic ionic, and gating currents using a variety of Markov schemes. The nine state diagram 


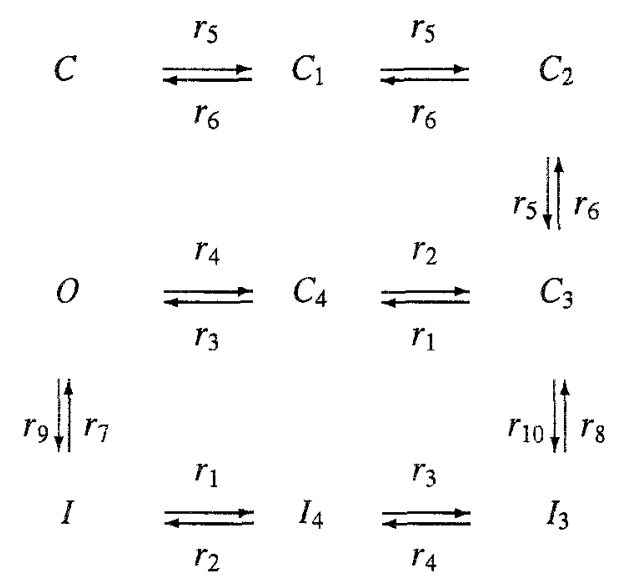

was found to be optimal by maximum likelihood criteria. The voltage-dependence of the transition rates was assumed to be a simple exponential function of voltage (Eq. 9). To complement the detailed sodium model of Vandenberg and Bezanilla, we also examined the six state scheme for the squid delayed rectifier used by Perozo and Bezanilla $(1990,1992)$

$$
\begin{aligned}
& c \stackrel{r_{1}}{\rightleftharpoons} C_{1} \stackrel{r_{3}}{\rightleftharpoons} \underset{r_{4}}{\rightleftharpoons} C_{2} \\
& O \stackrel{r_{6}}{\rightleftharpoons} C_{4} \stackrel{r_{4}}{\rightleftharpoons} C_{3}
\end{aligned}
$$

where again rates were described by a simple exponential function of voltage (Eq. 9).

Magnesium Block. In the case of the N-methyl-Daspartate (NMDA) receptor-gated channel there is a marked voltage-dependency in the presence of extracellular magnesium (Mayer et al., 1984; Nowak et al., 1984). Magnesium acts by a direct block of the channel's ion pore, rather than by introducing a new voltage-dependent conformational change (Jahr and Stevens, 1990a, b). Consequently, the model for this channel can be simplified by assuming magnesium- and voltage-independent gating properties of the channel may be treated independently of the magnesium block. Furthermore, since the kinetics of the magnesium block are extremely rapid, the gating by magnesium can be considered to be at equilibrium. Thus, Jahr and Stevens (1990b) found that for physiological magnesium concentrations, the NMDA receptor-mediated current can be described by a voltage- and magnesium-independent kinetics of the receptor, whereas the dependence in voltage can be integrated in a gating function that multiplies the conductance. This gating function is

$$
\begin{aligned}
& f_{N M D A}\left(V, M g^{2+}\right)= \\
& 1 /\left[1+\exp (-0.062 V)\left(\left[M g^{2+}\right]_{o} / 3.57\right)\right]
\end{aligned}
$$

where $\left[M g^{2+}\right]_{0}$ is the extracellular $M g^{2+}$ concentration, and the constants were determined empirically based on single-channel studies. The total NMDA conductance is then proportional to the product of this expression and the fraction of channels in the open state.

\subsection{Models of Ligand-Gated Channels}

The presence of one or more receptor sites on a channel confers a second important class of gating properties, that of ligand-gating. A principle class of ligand-gated channels is those activated directly by neurotransmitter. Ligand gating also commonly occurs through secondary agonists, such as the glycine activation of the NMDA receptor, or through secondmessengers, such as calcium, G-proteins, or cyclic nucleotides.

For a ligand-gated channel, transition rates between unbound and bound states of the channel depend on the concentration of ligand

$$
L+S_{i} \stackrel{r_{i j}}{\rightleftharpoons} S_{j i}
$$

where $L$ is the ligand, $S_{i}$ is the unbound state, $S_{j}$ is the bound state (sometimes written $S_{i} L$ ), $r_{i j}$ and $r_{j i}$ are rate constants as defined before.

The same reaction can be rewritten as:

$$
S_{i} \stackrel{r_{i j}([L])}{\underset{r_{j i}}{\rightleftarrows}} S_{j}
$$

where $r_{i j}([L])=[L] r_{i j}$ and $[L]$ is the concentration of ligand. Written in this form, (21) is equivalent to (5). Ligand-gating schemes are generally equivalent 
to voltage-gating schemes, although the functional dependence of the rate on $[L]$ is simple compared to the voltage-dependence discussed in the preceding section.

Alpha Functions. A very simple function which has been used to describe transmitter-gated responses is the alpha function, originally introduced by Rall (1967)

$r\left(t-t_{0}\right)=\frac{\left(t-t_{0}\right)}{\tau_{1}} \exp \left[-\left(t-t_{0}\right) / \tau_{1}\right]$

The function gives a stereotyped waveform for the time course of the postsynaptic current following a presynaptic spike occurring at time $t=t_{0} \cdot \tau_{1}$ is the time constant of the alpha function. Alpha functions often provide approximate fits for many synaptic currents, and have been widely used for computing synaptic currents in neural models (see e.g. Koch and Segev, 1989).

Markov Models of Ligand-Gated Channels. Although it was originally chosen empirically, without an underlying microscopic interpretation, it is also possible to derive the alpha function from a particular transmitter gating scheme under several restricted assumptions (Appendix B). We outline here detailed and elementary kinetic schemes that can be used more generally to describe postsynaptic currents. State diagrams are written to represent the conformational changes of the synaptic channel. The most elementary state diagram for a ligand-gated channel is

$$
C \stackrel{r_{1}([L])}{\rightleftarrows} \mathrm{r}
$$

where $C$ and $O$ represent the closed and open states of the channel and $r_{1}([L])$ and $r_{2}$ are the associated rate constants.

In some cases, the presence of one or several desensitized states must be introduced to account for the time-dependent properties of the channel. Desensitized states, as they are called in the receptor kinetic literature, are equivalent to the inactivated states of voltage-dependent channels. The addition of a single desensitized state to the elementary scheme gives:

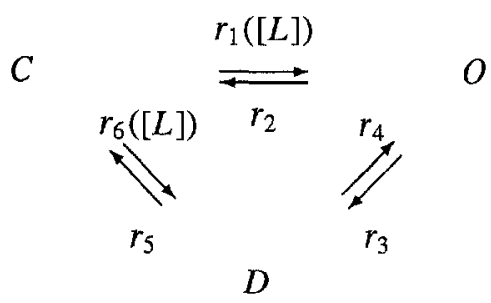

Here $D$ represents the desensitized state of the channel and $r_{1} \ldots r_{6}$ are the associated rate constants. Slightly simpler 3 -state models result when some transitions are excluded.

Detailed, multi-state kinetic schemes have been derived for a wide range of transmitter-gated receptors, including the nicotinic acetylcholine receptor (e.g. Sakmann, 1992), the non-NMDA (e.g. Raman and Trussell, 1992; Standley et al., 1993) and NMDA subtypes of glutamate receptor (e.g. Clements and Westbrook, 1991; Lester and Jahr, 1992) and the $\gamma$ aminobutyric acid $\left(\mathrm{GABA}_{A}\right)$ receptor (e.g. MacDonald and Twyman, 1992). As an example of such a detailed model, we considered a fast, non-NMDA glutamate receptor (we do not distinguish here between the various types of non-NMDA glutamate receptors, which we refer to as $\alpha$-amino-3-hydroxy-5-methyl-4isoxazoleproprionic acid/kainate or AMPA/kainate). The AMPA/kainate glutamate receptor of the locust muscle was characterized by Standley et al. (1993) using single-channel recording techniques, and several models were tested to account for the observed gating kinetics. They found that their data was fit by the six-state scheme

$$
\begin{aligned}
& C \stackrel{r_{1}([L])}{\rightleftharpoons} C_{2} \stackrel{r_{3}([L])}{\underset{r_{4}}{\rightleftarrows}} C_{2} \stackrel{r_{5}}{\underset{r_{6}}{\rightleftharpoons}} O \\
& r_{7}([L]) \| r_{8} \\
& D_{1} \stackrel{r_{9}([L])}{\underset{r_{10}}{\rightleftharpoons}} D_{2}
\end{aligned}
$$

where $C$ is the unbound closed state, $C_{1}$ and $C_{2}$ are respectively the singly- and doubly-bound closed states, $O$ is the open state, and $D_{1}$ and $D_{2}$ are respectively the desensitized singly- and doubly-bound states. 
Pulse Models and Exact Expression for the Postsynaptic Current. A Markov kinetic scheme describes the movement between different conformational states $\left(S_{1} \ldots S_{n+1}\right)$ of a protein by a set of $n$ first-order differential equations. Generally, such a system admits an exact solution if all rates, $r_{i j}$, are constant. The solution for a Markov system with $(n+1)$ states is a sum of $n$ exponentials:

$s_{i}(t)=s_{\infty_{i}}+\sum_{j=1}^{n} K_{i j} \exp \left(-t / \tau_{j}\right)$

where the coefficients $s_{\infty_{i}}, K_{i j}$ and the time constants $\tau_{j}$ are functions of the rate constants $r_{i j}$ and the coefficients $K_{i j}$ also depend on the initial conditions.

As described above, channel gating by either ligand or voltage leads to transition rates that vary as the electrical or chemical environment of the channel changes over time. Therefore, in order to apply an analytic solution, conditions must be found in which these dynamic variables can be considered constant.

In a previous paper (Destexhe et al., 1994b), we gave an analytic expression for a postsynaptic current from a two-state ligand-gated channel (see also Staubuli et al., 1992). The analysis was based on the assumption that the channel experienced transmitter concentrations that were piecewise constant, such as during a brief pulse. There are separate solutions during the pulse, when the concentration of the transmitter is high, and after the pulse, when the concentration of the transmitter returns to zero. The use of a pulse-shaped transmitter concentration was justified in reference to experiments using the "liquid filament" technique, which showed that $1 \mathrm{~ms}$ pulses of $1 m M$ glutamate reproduced PSC's in membrane patches that were quite similar as those recorded in the intact synapse (Hestrin, 1992; Colquhoun et al., 1992; Standley et a1., 1993). The same considerations were applied more generally here. In addition to the two-state model, we examined pulse-based analytic solutions for three-state channel models (Eq. 24). These expressions are given in Appendix A.

Pulse models were based on the delivery of transmitter using a procedure identical to the one described before (Destexhe et al., 1994b). Following the arrival of an action potential in the presynaptic compartment, a rectangular pulse of transmitter is triggered in the synaptic cleft. The pulse is started when the presynaptic voltage crosses $0 \mathrm{mV}$ and the postsynaptic con- ductance is calculated using the analytic expressions given in Appendix A.

Analytic solutions are also applicable for calculating the time course of a voltage-dependent channel following a voltage clamp. A similar approach was followed by Goldman and Hahin (1979) for the sodium current. Although we give the general solution for voltage-dependent and ligand-dependent cases in Appendix A, they were not used explicitly for the voltage-dependent solutions here. They can be applied in principle for estimating the parameters of first- and second-order models from voltage-clamp data (Goldman and Hahin, 1979).

During a numerical simulation, one can make the approximation that voltage and ligand concentration are constant during any small time step, $d t$. Transition rates can then be calculated at $t=t_{0}$ as functions of $V\left(t_{0}\right)$ and $[L]\left(t_{0}\right)$. The state at $t=t_{0}+d t$ is calculated with these constant rates using the equations described in Appendix A.

Second Messenger Gating. Some neurotransmitters do not bind directly to the ion channel, but modulate the channel through an intracellular messenger, which links the activated receptor to the opening or closing of an ion channel. We developed such a kinetic model for a class of neurotransmitters linked to the activation of a G-protein pathway involved in the gating of a $K^{+}$channel (see Results). We compared this model with a much simplified model for such a response. The simple model assumed that following transmitter binding to postsynaptic receptors, a G-protein was activated in a pulse-like fashion for a fairly long duration (50-100 $\mathrm{ms}$ ). In this case, the model of G-protein-mediated postsynaptic response reduced to the same functional expression as Eq. (20) and the considerations applied to transmitter-gated channels also applied to the G-protein model.

\subsection{Integration of Kinetic Models into Neural Models}

Kinetic models of ion channels and other proteins are coupled through various types of interactions, which can be expressed by equations governing the electrical and chemical states of the cell.

Electric Interactions. Using the equivalent circuit approach, the general equation for the membrane 
potential of a single isopotential "compartment" is:

$$
C_{m} \frac{d V}{d t}=\sum_{k=1}^{n} I_{k}
$$

where $V$ is the membrane potential, $C_{m}$ is capacitance of the membrane, and $I_{k}$ are the contributions of all channels of one type to the current across a particular area of membrane. Only single compartments were simulated, but the same approach could be extended to multiple compartments using the method of equivalent electrical circuits (Koch and Segev, 1989). In this case, a compartment may represent a small cylinder of dendritic or axonal process. We assumed that (a) the membrane compartment contained a sufficiently large number of channels of each type $k$ for Eq. (4) to hold, and (b) there was a single open or conducting state, $O_{k}$, for each channel type with maximum single-channel conductance, $\gamma_{k}$. Then, to first approximation, each $I_{k}$ can be calculated assuming a linear $I-V$ relationship, giving the familiar Ohmic form

$$
I_{k}=\bar{g}_{k} o_{k}\left(V-E_{k}\right)
$$

where $o_{k}$ is the fraction of open channels, $\bar{g}_{k}$ is the maximum conductance and $E_{k}$ is the apparent equilibrium (reversal) potential. $\bar{g}_{k}$ is the product of the single-channel conductance and the channel density, $\bar{g}_{k}=\gamma_{k} \rho_{k}$.

The assumption of an ohmic $I-V$ relation breaks down when an extreme concentration gradient exists for a permeable ion. The Goldman-Hodgkin-Katz current equation is more appropriate to model nonequilibrium situations for which this breakdown can occur (Hille, 1992). This equation was used to describe $\mathrm{Ca}^{2+}$ currents, namely

$$
\begin{aligned}
I_{k}= & \bar{p}_{k} o_{k} \frac{V z^{2} F^{2}}{R T} \\
& \frac{\left[C a^{2+}\right]_{i}-\left[C a^{2+}\right]_{o} \exp (-z F V / R T)}{1-\exp (-z F V / R T)}
\end{aligned}
$$

where $\bar{p}_{k}$ is maximum permeability, $z=2$ is the valence of $\mathrm{Ca}^{2+}$, and $\left[\mathrm{Ca}^{2+}\right]_{i}$ and $\left[\mathrm{Ca}^{2+}\right]_{o}$ are calcium concentrations on the inside and outside of the membrane respectively, and $F$ is Faraday's constant, $R$ is the gas constant, and $T$ is the absolute temperature.
Influx and Efflux of Ions. Calcium acts as ligands for many channels and proteins. In order to track the concentration of ions such this, standard chemical kinetics can be used. The contribution of calcium channels to the free $\mathrm{Ca}^{2+}$ inside the cell was calculated as

$$
\frac{d\left[C a^{2+}\right]_{i}}{d t}=\frac{-I_{C a}}{z F A d}
$$

where $A$ is the membrane area, $d=0.1 \mu \mathrm{m}$ is the depth of an imaginary submembrane shell, and the other constants are as given for Eq. (29). Removal of intracellular $\mathrm{Ca}^{2+}$ was driven by an active pump obeying Michaelis-Menten kinetics (see Destexhe et al., 1993a):

$$
\mathrm{Ca}_{i}^{2+}+\mathrm{P} \underset{c_{2}}{\stackrel{c_{1}}{\rightleftarrows}} \mathrm{CaP} \stackrel{c_{3}}{\longrightarrow} \mathrm{P}+\mathrm{Ca}_{o}^{2+}
$$

where $P$ represents the $C a^{2+}$ pump, $C a P$ is an intermediate state, $\mathrm{Ca}_{0}^{2+}$ is the extracellular $\mathrm{Ca}^{2+}$ and $c_{1}, c_{2}$, and $c_{3}$ are rate constants as indicated. $C a^{2+}$ ions have a high affinity for the pump $P$, whereas extrusion of $\mathrm{Ca}^{2+}$ follows a slower process (Blaustein, 1988). The extrusion process was assumed to be fast (milliseconds). Diffusion was not calculated.

\subsection{Fit of Models to Whole-Cell Recordings}

Postsynaptic currents resulting from a presynaptic action potential were fit to PSC's obtained by whole-cell voltage-clamp recordings in hippocampal slices. For AMPA/kainate receptors, averaged PSC's recorded from mossy fiber synapses in CA3 pyramidal cells were provided by Z. Xiang and T. Brown (see Xiang et al,, 1992). Averaged PSC's of NMDA receptors were provided by N.A. Hessler and R. Malinow (see Hessler et al., 1993). For GABA receptors, $\mathrm{GABA}_{A}$ and GABA $_{B}$ PSC's recorded from dentate granule cells were provided by T. Otis and I. Mody (see Otis and Mody, 1992; Otis et al., 1993).

Models were fit to the experimental waveforms using a simplex least squares fitting algorithm (see Press et al., 1986). In the case of first- or secondorder models, the time course of the PSC could be obtained as an analytical expression using the procedure described above (see also Appendix A). This expression was fit to the data using the standard version 


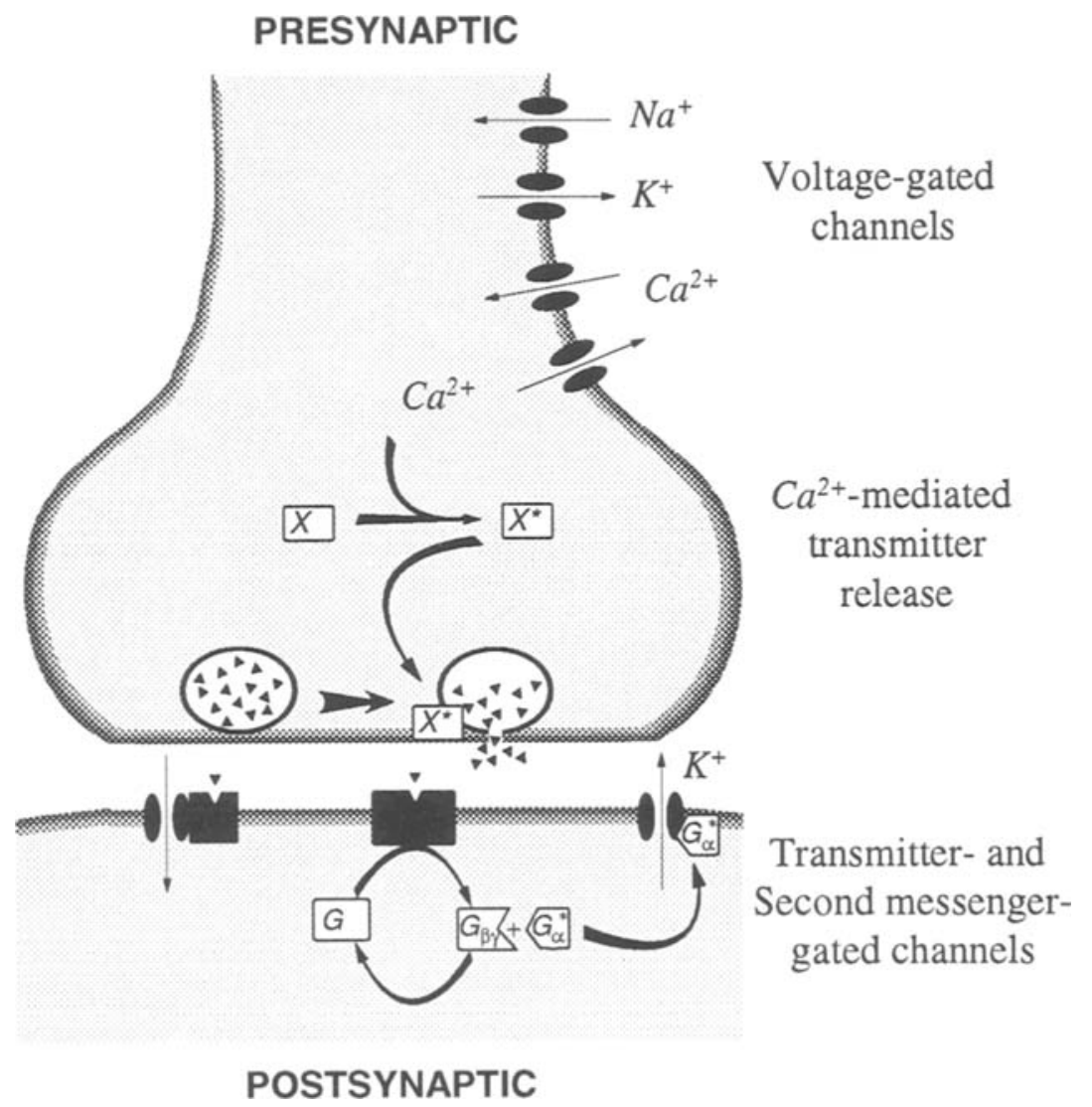

Fig. 1. Schematic diagram of the kinetic schemes used for modeling synaptic transmission. The voltage-gated channels depolarize the presynaptic terminal; $\mathrm{Ca}^{2+}$ enters the cytosol via a high-threshold $\mathrm{Ca}^{2+}$ current activated during the action potential; $\mathrm{Ca}^{2+}$ ions bind to a calcium-binding protein $(X)$ and produces an active form $\left(X^{*}\right) ; X^{*}$ promotes the exocytosis of synaptic vesicles and release of transmitter molecules (triangles) into the synaptic cleft. The transmitter can bind to a postsynaptic receptor-ionophore complex (left), or to a secondmessenger-linked postsynaptic receptor (right). In the latter case, the receptor catalyzes the formation of an activated G-protein alpha subunit $\left(G_{\alpha}^{*}\right)$, which directly gates the opening of a potassium channel. All of the steps in this process are described by simple kinetic models in the text.

of the simplex algorithm. In the case of higher-order models, a direct fit of the kinetic model was more efficient. In this case, the kinetic equations of the model were solved numerically using an Euler integration scheme for each iteration of the simplex procedure. All possible first- and second-order kinetic schemes were considered when fitting models to these data.

\section{Results}

The kinetic models outlined in the Methods allow voltage- and ligand-gated ion channels to be described within the same framework as that for the molecular interactions underlying signal transduction and cellular biochemistry. Here, we use this common kinetic framework to construct a simple model of synaptic transmission. The different processes incorporated into the model are illustrated in Fig. 1.

We begin with both detailed and simple kinetic models of voltage-dependent ion channels, using the generation of a classical sodium-potassium action potential as an example. We then present a kinetic model of transmitter release incorporating calcium entry and efflux and a simplified calcium-triggered signal cascade leading to vesicle fusion. The outcome of the release process is the time-varying concentration of neurotransmitter in the synaptic cleft, which in turn gates postsynaptic channels. Both traditional "fast" transmitter-gated ion channels and "slow," modulatory, conductances are considered. For both fast and slow synaptic responses, we compare elementary and detailed kinetic schemes to experimental recordings, 
A

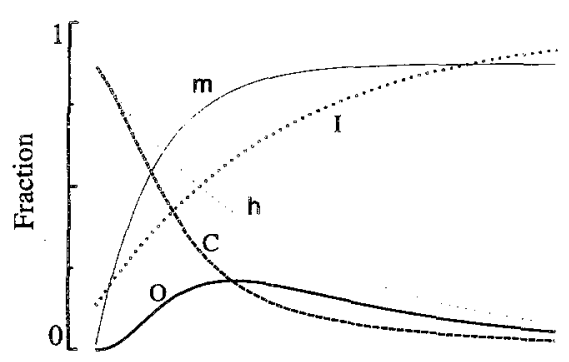

B

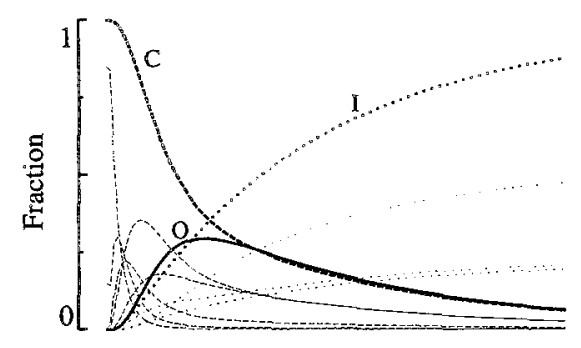

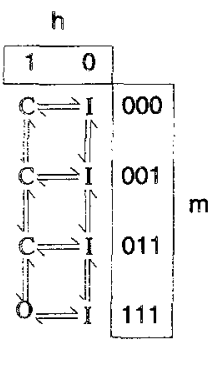

c

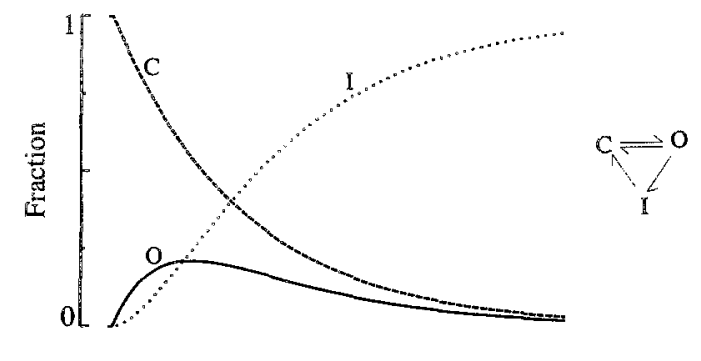

$1 \mathrm{msec}$

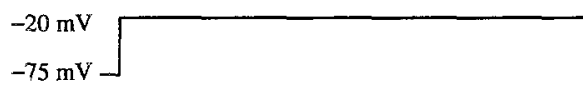

D

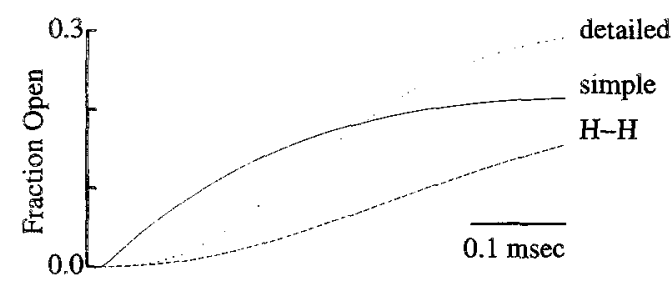

Fig. 2. Three kinetic models of a squid axon sodium channel produce qualitatively similar conductance time courses. A voltage-clamp step from rest, $V=-75 \mathrm{mV}$, to $V=-20 \mathrm{mV}$ was simulated. The fraction of channels in the open state (O, solid lines), closed states (C, dashed lines), and inactivated states ( $I$, dotted lines) are shown for the Hodgkin-Huxley model, a detailed markov model, and a simple Markov model. A. An equivalent Markoy scheme for the Hodgkin-Huxley model is shown (right insert, Eq. 14). Three identical and independent activation gates ( $m$, thin solid line) gives a form with 3 closed states (corresponding to 0,1 , and 2 activated gates) and one open state ( 3 activated gates). The independent inactivation gate, $(h$, thin dotted line) adds 4 corresponding inactivated states. Voltage-dependent transitions were calculated using the original equations and constants of Hodgkin and Huxley (1952). B. The detailed Markov model of Vandenberg and Bezanilla (1991) (Eq. 17; $a_{1}=11490 \mathrm{~s}^{-1}, b_{1}=59.19 \mathrm{mV}, a_{2}=8641 \mathrm{~s}^{-1}, b_{2}=-5860 m V, a_{3}=31310 \mathrm{~s}^{-1}, b_{3}=17.18 \mathrm{mV}$, $a_{4}=2719 s^{-1}, b_{4}=-51.54 m V, a_{5}=33350 s^{-1}, b_{5}=74.58 m V, a_{6}=1940 s^{-1}, b_{6}=-21.03 m V, a_{7}=863.1 s^{-1}, b_{7}=27050 m V$, $\left.a_{8}=1538 s^{-1}, b_{8}=27050 m V, a_{9}=7.992 s^{-1}, b_{9}=-27.07 m V, r_{10}=r_{8} r_{9} / r_{7}\right)$. Individual closed and inactivated states are shown (thin lines) as well as the sum of all 5 closed and all 3 inactivated states (thick lines). C. A simple three-state Markov model fit to approximate the detailed model (Eq. 32; $a_{1}=1500 s^{-1}, a_{2}=200 s^{-1}, a_{4}=150 s^{-1}, b=5 m V, c_{1}=c_{2}=-27 m V, c_{4}=-65 m V, r_{3}=3000 s^{-1}$ ). D. Comparison of the time course of open channels for the three models on a faster time scale shows differences immediately following a voltage step. The Hodgkin-Huxley model (dashed line) and detailed Markov modeled (solid line) give smooth, multi-exponential rising phases, while the simple Markov model (dotted line) gives a single exponential rise with a discontinuity in the slope at the beginning of the pulse.

and provide analytic expressions for PSC's based on further simplifying assumptions.

\subsection{Voltage-Gated Ion Channels}

Voltage-dependent channels can be modeled comprehensively using kinetic schemes in which some of the transition rates vary with membrane potential. The minimal kinetic model for a voltage-dependent channel is the first order scheme consisting of just one closed or non-conducting state and one open or conducting state. The two-state description is adequate to fit the behavior of some channels (see e.g., Labarca et al., 1980; Yamada et al., 1989; Borg-Graham, 1991), but for most channels more complex models must be considered. Many models of sufficient complexity are capable of fitting any limited set of experimental data. To demonstrate this, we compared three alternative models of the fast sodium channel of the squid axon (Fig. 2).

First, the original quantitative description of the squid giant axon sodium conductance given by Hodgkin and Huxley (1952) was reproduced (Fig. 2A). The Hodgkin-Huxley model ( $\mathrm{H}-\mathrm{H}$ model) had four independent gates each undergoing transitions between two states with first-order kinetics as 
described by Eq. (12). Three identical gates, $m$, represent activation and one, $h$, inactivation, leading to the familiar form for the conductance, $g_{N a} \propto m^{3} h$ (Eq. 13).

Since the work of Hodgkin and Huxley, the behavior of the sodium channel of the squid axon has subsequently been better described in studies using Markov kinetic models. To illustrate the nature of these studies, we simulated the detailed sodium channel model of Vandenberg and Bezanilla (1991) (Eq. 17, Fig. 2B). This particular nine-state model was selected to fit not only the measurements of macroscopic ionic currents available to Hodgkin and Huxley, but also recordings of single channel events and measurements of currents resulting directly from the movement of charge during conformational changes of the protein (socalled gating currents).

Finally, we created a very simple Markovian sodium channel model (Fig. 2C). The scheme was chosen to have the fewest possible number of states (three) and transitions (four) while still being capable of reproducing the essential behavior of the more complex models. The form of the state diagram was based on the looped three-state model (Eq. 16) with several transitions eliminated to give an irreversible loop (Bush and Sejnowski, 1991)

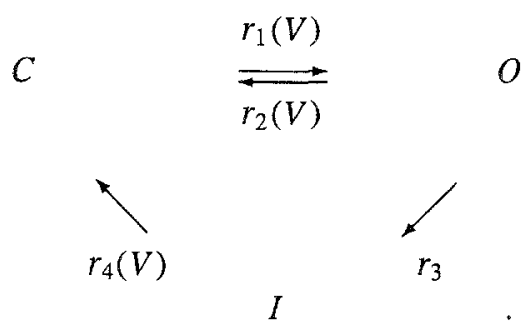

The model incorporated voltage-dependent opening, closing, and recovery from inactivation, while inactivation was voltage-independent. For simplicity, neither opening from the inactivated state nor inactivation from the closed state were permitted. Although there is clear evidence for occurrence of the latter (Horn et al., 1983) it was unnecessary under the conditions of our simulations.

It is common for detailed Markov models of voltage-gated channels to assume that the voltagedependence of all rates takes a simple exponential form (Eq. 9; e.g. Chabala, 1984; Vandenberg and Bezanilla, 1991; Perozo and Bezanilla, 1990; Harris et al., 1981). However, as discussed in the Methods, the interactions of a channel pro- tein with the membrane field might be expected to yield a rather more complex dependency (Stevens, 1978; Neher and Stevens, 1978; Andersen and Koeppe, 1992; Clay, 1989). Significantly, it has been shown that the number of states needed by a model to reproduce the voltage-dependent behavior of a channel may be reduced by adopting functions that saturate at extreme voltages (Keller et al., 1986; Clay, 1989; Chen and Hess, 1990; Borg-Graham, 1991). Therefore, we described the voltage-dependent transition rates of the simple sodium channel model by the sigmoidal function

$$
r_{i}(V)=\frac{a_{i}}{1+\exp \left[-\left(V-c_{i}\right) / b_{i}\right]}
$$

which can be obtained from Eq. (8) by considering nonlinear as well as linear components of the voitage dependence of the free energy barrier between states. The constant $a_{i}$ sets the maximum transition rate, $b$ sets the steepness of the voltage-dependence, and $c_{i}$ sets the voltage at which the half-maximal rate is reached. Through explicit saturation, Eq. (33) effectively incorporates voltage-independent transitions that become rate-limiting at extreme voltage ranges (Keller et al., 1986, Vandenberg and Bezanilla, 1990, Chen and Hess, 1990), eliminating the need for additional closed or inactivated states (see discussion by Chen and Hess, 1990). We constrained all $b_{i}=b$ and $c_{1}=c_{2}$ to yield a model consisting of nine total parameters.

The response of the three sodium channel models to a voltage-clamp step from rest $(-75 m \mathrm{~m})$ was simulated (Fig. 2). For all three models, closed states were favored at hyperpolarized potentials. Upon depolarization, forward (opening) rates sharply increased while closing (backward) rates decreased, causing a migration of channels in the forward direction toward the open state. The three closed states in the Hodgkin-Huxley model and the five closed states in the detailed (Vandenberg-Bezanilla) model gave rise to the characteristic sigmoidal shape of the rising phase of the sodium current (Fig. 2D). In contrast, the simple model, with a single closed state, produced a first-order exponential response to the voltage step. As expected, the addition of one or more closed states to the simple model led to a progressively more sigmoidal rising phase (not shown). 
Even though the steady-state behavior of the Hodgkin-Huxley model of the macroscopic sodium current is remarkably similar to that of the microscopic Markov models (Marom and Abbott, 1994), the relationship between activation and inactivation is different. First, in the Hodgkin-Huxley model, activation and inactivation are kinetically independent. This independence has been shown to be untenable on the basis of gating and ion current measurements in the squid giant axon (Armstrong, 1981; Bezanilla, 1985). Consequently, Markov models that are required to reproduce gating currents, such as the Vandenberg-Bezanilla model examined here, require schemes with coupled activation and inactivation. Likewise, in the simple model, activation and inactivation were strongly coupled due to the unidirectional looped scheme (Eq. 32 ), so that channels were required to open before inactivating and could not reopen from the inactivated state before closing.

Second, in the Hodgkin-Huxley and VandenbergBezanilla models, inactivation rates are slow and activation rates fast. In the simple Markov model, the situation was reversed, with fast inactivation and slow activation. At the macroscopic level modeled here, these two relationships gave rise to similar times course for open channels (Fig. 2A-C; see Andersen and Koeppe, 1992). However, the two classes of models make distinct predictions when interpreted at the microscopic (single-channel) level. Whereas the Hodgkin-Huxley and Vandenberg-Bezanilla models predict the latency to first channel opening to be short and channel open times to be comparable to the time course of the macroscopic current, the simplified Markov model predicts a large portion of first channel openings to occur after the peak of the macroscopic current and to have open times much shorter than its duration. Single channel recordings have confirmed the latter prediction (Sigworth and Neher, 1980; Aldrich et al., 1983; Aldrich and Stevens, 1987).

Despite the significant differences in their complexity and formulation, the three models of the sodium channel that we investigated all produced very comparable action potentials and repetitive firing when combined with appropriate delayed-rectifier potassium channel models (Fig. 3). None of the potassium channel models had inactivation. The main difference was in the number of closed states, from six for the detailed Markov model of Perozo and
Bezanilla (1989; Eq. 18), to four for the original (Hodgkin and Huxley, 1952) description of the potassium current, to just one for a minimal model (Eq. 10) with rates of sigmoidal voltage dependence (Eq. 33).

\subsection{Model of Transmitter Release}

The goal of this section was to model the link between presynaptic action potentials and the release of transmitter onto postsynaptic receptors. We modeled calcium-induced release along the lines of the model introduced recently by Yamada and Zucker (1992). There is ongoing debate concerning the exact mechanisms whereby $\mathrm{Ca}^{2+}$ enters the presynaptic terminal, the specific proteins with which $\mathrm{Ca}^{2+}$ interacts, and the detailed mechanisms leading to exocytosis. For the purposes of our simple model, we assumed that: (a) upon invasion by an action potential, $\mathrm{Ca}^{2+}$ enters the presynaptic terminal due to the presence of a high-threshold $\mathrm{Ca}^{2+}$ current; (b) $\mathrm{Ca}^{2+}$ activates a calcium-binding protein which promotes release by binding to the transmitter-containing vesicles; (c) an inexhaustible supply of "docked" vesicles are available in the presynaptic terminal, ready to release; (d) the binding of the activated calcium-binding protein to the docked vesicles leads to the release of $n$ molecules of transmitter in the synaptic cleft. This process is modeled here as a first-order process with a stoechiometry coefficient of $n$.

The sodium and potassium channels underlying the action potential were described by the simple (three and two state, respectively) Markov models described in the preceding section. Calcium entry into the presynaptic terminal was modeled by a high-threshold $\mathrm{Ca}^{2+}$ current, using the same two-state Markov scheme and voltage-dependent rate functions as the potassium current (Eqs. 10 and 33). The parameters of the rate functions were chosen to approximate the L-type calcium channels described in McCormick and Huguenard (1992). Removal of intracellular $\mathrm{Ca}^{2+}$ was driven by an active pump (see Methods).

The calcium-induced cascade leading to the release of transmitter (Fig. 1) was described by the following kinetic scheme:

$$
4 \mathrm{Ca}^{2+}+X \underset{k_{u}}{\stackrel{k_{b}}{\rightleftarrows}} X^{*}
$$


A

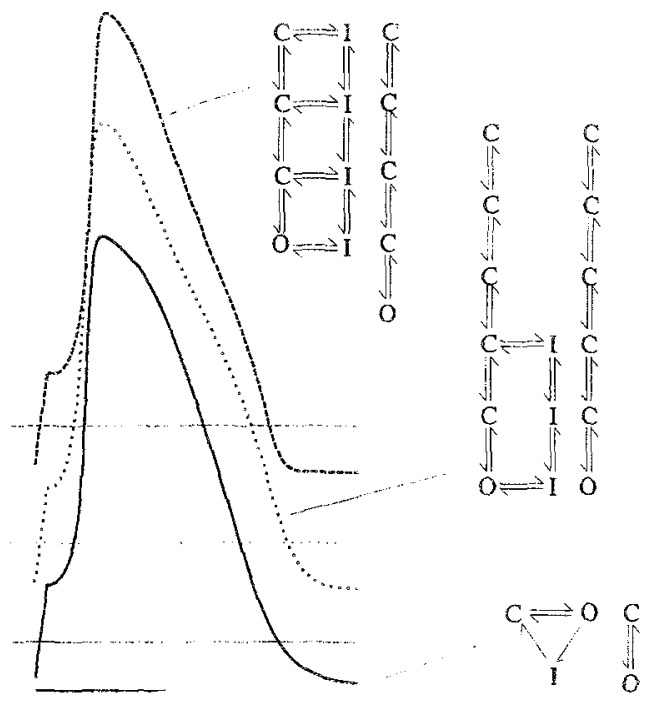

$2 \mathrm{~ms}$

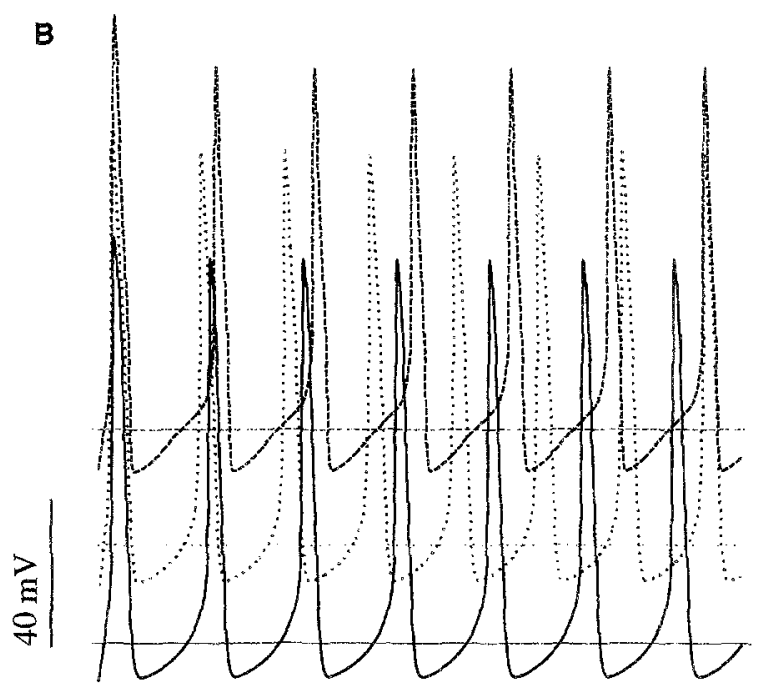

$20 \mathrm{~ms}$

Fig. 3. Similar action potentials produced using three different kinetic models of squid fast sodium and delayed rectifying potassium channels. A. Single action potentials in response to $0.2 \mathrm{~ms}, 2 \mathrm{nA}$ current pulse are elicited at similar thresholds and produce similar waveforms using three different pairs of kinetic models: Hodgkin-Huxley (dashed line; Hodgkin and Huxley, 1952), detailed Markov (dotted line; Vanderberg and Bezanilla, 1991; Perozo and Bezanilla, 1989), and simple Markov (solid line). B. Repetitive trains of action potentials elicited in response to sustained current injection $(0.2 \mathrm{nA})$ have slightly different frequencies. Sodium channels were modeled as described in Fig. 2 . The detailed Markov potassium channel model had 6 states (Perozo and Bezanilla, 1989) (Eq. 18; $a_{1}=484.5 s^{-1}, b_{1}=112 m V, a_{2}=19.23 s^{-1}$, $\left.b_{2}=-8.471 \mathrm{mV}, a_{3}=1757 \mathrm{~s}^{-1}, b_{3}=25.83 \mathrm{mV}, a_{4}=569 \mathrm{~s}^{-1}, b_{4}=-491.0 \mathrm{mV}, a_{5}=672.9 \mathrm{~s}^{-1}, b_{5}=212 \mathrm{mV}, a_{6}=784.4, b_{6}=0\right)$. The simple Markov potassium channel had 2 states (Eq. 10 with rates given by the sigmoid function of Eq. $33 ; a_{1}=100 s^{-1}, a_{2}=240 s^{-1}$, $b=5 m V, c_{1}=c_{2}=-27 m V$. 


$$
X^{*}+V_{e} \stackrel{k_{1}}{\underset{k_{2}}{\rightleftarrows}} V_{e}^{*} \stackrel{k_{3}}{\longrightarrow} n L .
$$

Calcium ions bind to a calcium-binding protein, $X$, with a cooperativity factor of 4 (see Augustine and Charlton, 1986; and references therein), leading to an activated calcium-binding protein, $X^{*}$ (Eq. 34). The associated forward and backward rate constants are $k_{b}$ and $k_{u} . X^{*}$ then reversibly binds to transmittercontaining vesicles, $V_{e}$, with corresponding rate constants $k_{1}$ and $k_{2}$ (Eq. 35). The last step of this reaction, governed by rate constant $k_{3}$, represents the (irreversible) release of $n$ molecules of transmitter, $L$, from the activated vesicles into the synaptic cleft. The values of the parameters in this reaction scheme were based on previous models and measurements (Yamada and Zucker, 1992).

The concentration of the liberated transmitter in the synaptic cleft, $[L]$, was assumed to be uniform and cleared by processes of diffusion, uptake or degradation. These contributions were modeled by the first order reaction:

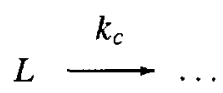

where $k_{c}$ is the rate constant for clearance of $L$.

Figure 4 shows a simulation of transmitter release triggered by a presynaptic action potential. Injection of a short current pulse into the presynaptic terminal elicited a single action potential (Fig. 4A). The depolarization of the action potential activated L-type calcium channels, producing a rapid influx of calcium. The elevation of intracellular $\left[\mathrm{Ca}^{2+}\right]$ (Fig. 4B) was transient due to clearance by the active pump. The time-course of activated calcium-binding proteins and vesicles followed closely the time-course of the transient calcium rise (Fig. 4C). This resulted in a brief $(\approx 1 \mathrm{~ms})$ rise in transmitter concentration the synaptic cleft (Fig. 4D). The rate of transmitter clearance was adjusted to match the time course of transmitter release estimated from patch clamp experiments (Clements et al., 1992). Transmitter in the cleft could then bind and activate postsynaptic ligandgated channels producing a postsynaptic current and potential (Fig. 4E,F). The kinetics of the postsynaptic response are described below.
Simplification of the Release Process. Scrutiny of the time course of variables in the model of presynaptic release (Fig. 4) showed that the time course of the transmitter concentration $([L])$ followed grossly that of the presynaptic voltage. This was due to the fact that all intervening reactions occurred at relatively fast rates. Under the assumption that all species involved were near their equilibrium values, we were able to derive an expression for $[L]$ as a function of presynaptic voltage.

The stationary relationship between $[L]$ and presynaptic voltage was evaluated by simulating a voltage-clamp protocol. Figure 5 shows such a protocol in which the stationary concentration of $L$ is represented as a function of the value at which the presynaptic membrane is clamped. The critical step in this process was the activation of the presynaptic high-threshold $\mathrm{Ca}^{2+}$ current. This current begins to activate for depolarized values of the presynaptic membrane potential, around $-45 \mathrm{mV}$. For more depolarized potentials, the form of the pulse function depends on how the calcium current is obtained from the channel conductance (see Methods). When using the Goldman-Hodgkin-Katz equations, there was a bell-shaped relation, whereas using the standard Nernst equation for calculating the reversal potential, this function took the form of a sigmoid. The sigmoid form shown in Fig. 5 is actually much steeper than the activation function of the presynaptic highthreshold $\mathrm{Ca}^{2+}$ current. Taking this activation function to the 4th power (due to the cooperativity factor of the calcium-binding protein) gives a sigmoid function which is much closer to that of Fig. 5.

We fitted the steady-state values of transmitter concentration in Fig. 5 by a bell-shaped curve for the Goldman-Hodgkin-Katz model and a sigmoid function for the Nernst model. Both produced acceptable approximations, but in the following we present only the results from fitting the sigmoid form to the Nernst model:

$[L]\left(V_{p r e}\right)=\frac{L_{\max }}{1+\exp \left[-\left(V_{p r e}-V_{p}\right) / K_{p}\right)}$

where $L_{\max }$ is the maximal concentration of transmitter in the synaptic cleft, $V_{p r e}$ is the presynaptic voltage, $K_{p}=5 \mathrm{mV}$ gives the steepness and $V_{p}=2 m V$ sets the the value at which the function is half-activated. 

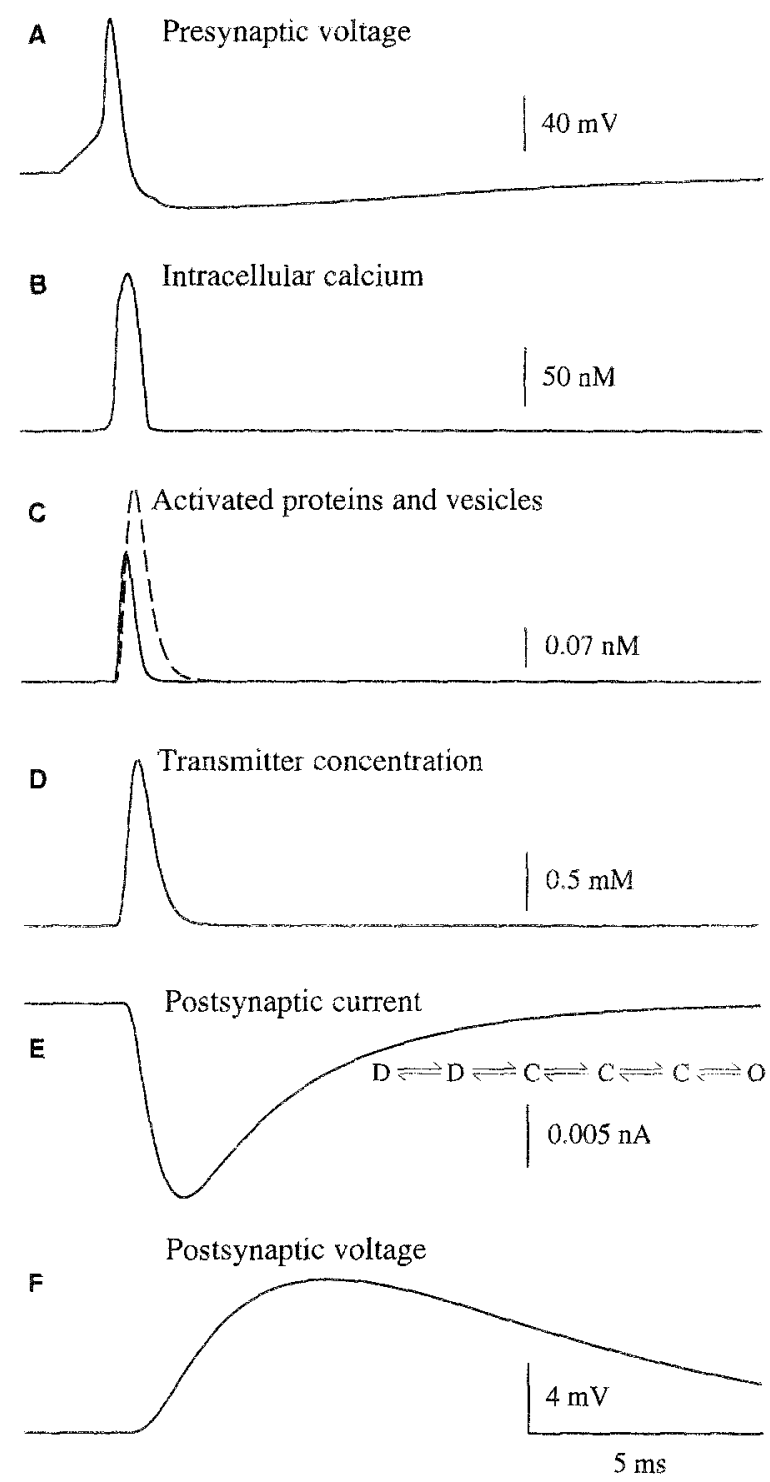

Fig. 4. Kinetic models of presynaptic release of glutamate and binding to transmitter-gated ion channels (AMPA/kainate receptors). A. A presynaptic action potential was elicited by injection of a $0.1 \mathrm{nA}$ current pulse lasting $2 \mathrm{~ms}$. Action potentials parameters for the simple kinetic models of sodium and potassium in Fig 3. B. Intracellular $\mathrm{Ca}^{2+}$ concentration in the presynaptic terminal. A high-threshold calcium current was also present and provided a transient calcium influx during the action potential. Removal was provided by an active calcium pump (see Eq. (31): $c_{2} / c_{1}=10^{-5} \mathrm{mM}, c_{3}=1 \mathrm{~s}^{-1}$ ). C. Relative concentration of activated calcium-binding protein $X^{*}$ (solid line; $k_{b}=10^{5} s^{-1} m M^{-4}, k_{u}=100 s^{-1}$ ) and activated vesicles $V_{e}^{*}$ (dashed line; $k_{1}=10^{6} s^{-1} m^{-1}, k_{2}=100 s^{-1}, k_{3}=4000 s^{-1}$, $V_{e}=0.01 \mathrm{mM}$ ). The maximal concentration of calcium-binding proteins was of $0.001 \mathrm{mM}$ and the number of transmitter molecules per vesicle was $n=10000$. D. Concentration of transmitter in the synaptic cleft $\left(k_{c}=10^{4} s^{-1}\right)$. The transmitter binds to AMPA/kainate receptors and opens the associated ion channel. E. Postsynaptic current produced by the gating of AMPA/kainate receptors according to kinetic scheme in Eq. (25) $\left(r_{1}=2 \cdot 10^{4} s^{-1} m M^{-1}, r_{2}=1300 s^{-1}, r_{3}=10^{4} s^{-1} m M^{-1}, r_{4}=2600 s^{-1}, r_{5}=900 s^{-1}, r_{6}=500 s^{-1}\right.$, $\left.r_{7}=10^{4} s^{-1} m M^{-1}, r_{8}=0.2 s^{-1}, r_{9}=2 s^{-1} m M^{-1}, r_{10}=0.1 s^{-1}, E_{r e v}=0 m V, \bar{g}_{A M P A}=1 n S\right)$. F. Excitatory postsynaptic potential in response to transmitter release (leakage conductance was $0.2 \mathrm{mS} / \mathrm{cm}^{2}$ and leakage reversal potential was $-70 \mathrm{mV}$ ). 


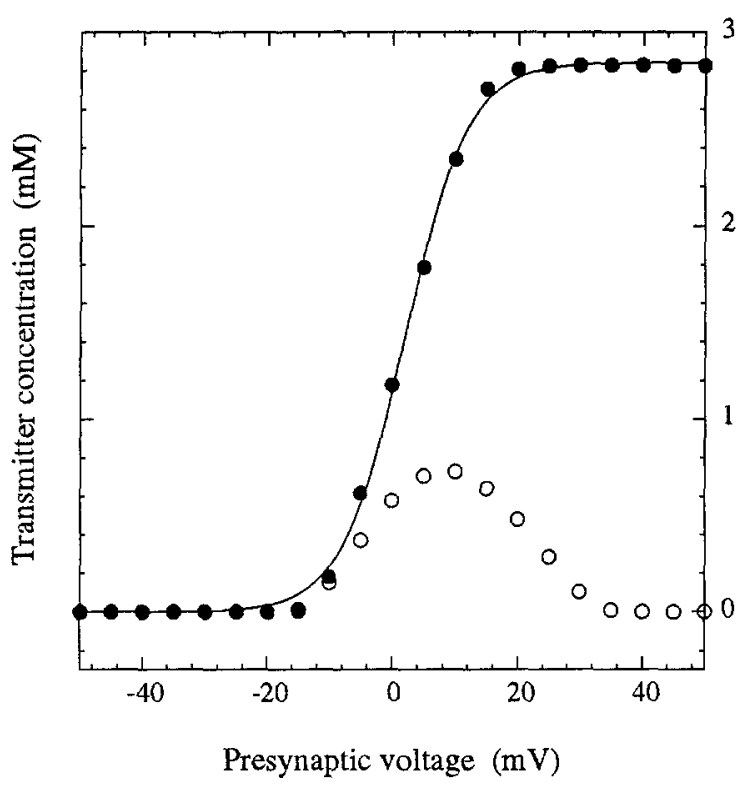

Fig. 5. Sigmoid relationship between transmitter concentration and presynaptic voltage. The kinetic model of presynaptic release was used with identical conditions as in Fig. 4. The presynaptic voltage was clamped at different values from -50 to $+50 \mathrm{mV}$. For each value, the model reached a steady state and the transmitter concentration was measured and represented here. The high-threshold calcium current was modeled as indicated in the text using either Goldman-Hodgkin-Katz equations (open circles) or the Nernst relation (filled circles). The sigmoid-like relationship observed for Nernst relation was fit using a least squares algorithm, leading to Eq. (37) with $L_{m a x}=2.84 m M, V_{p}=2 m V$ and $K_{p}=5 m V$.

As expected, this pulse function produced a transmitter time course very close to a pulse when the presynaptic terminal experienced transient depolarization by an action potential. We verified the efficiency of the pulse function by substituting it in a model in which synaptic interactions had been represented originally as conditionally-triggered pulses of transmitter (Destexhe et al., 1993b). This study described spindle oscillations in networks of cells with complex oscillatory properties. Although synaptic interactions were critical to the results of this model (Destexhe et al., 1993b), the same spindle oscillations were also found using expression (37) without adjusting the original values for any other parameters (data not shown).

One of the main advantages of using expression (37) is that it provides a very simple and smooth transformation between presynaptic voltage and transmitter concentration. This form, in conjunction with simple kinetic models of postsynaptic channels, provides a model of synaptic interaction based on autonomous differential equations with only one or two variables.

\subsection{Transmitter-Gated Ion Channels}

Postsynaptic transmitter-gated channels are a class of ligand-gated channel that can be represented using Markov kinetics, as outlined in the Methods section. A complete model for a postsynaptic response using Markov kinetics requires both the determination of a kinetic scheme for the channel and also specification of the time course of transmitter drives the response.

It would be very useful to have models that accurately captured the behavior of postsynaptic currents while remaining efficient to compute and/or amenable to analysis. There are many indications that the exact time course of transmitter in the synaptic cleft is not, under physiological conditions, a main determinant of the kinetics of postsynaptic responses at many synapses (e.g. Magelby and Stevens, 1972; Lester et al., 1990; Colquhoun et al., 1992; Clements et al., 1992). Thus, we explored two highly simplified models for transmitter time course. The first, described in the preceding section, was a function giving transmitter concentration directly as a function of presynaptic voltage. The use of this expression mimicked the behavior of the more elaborate release model while being much less expensive to calculate.

The second simplified description of transmitter time course was to consider transmitter concentrations occurring as a pulse. The chief advantage of the pulse method is that it allows the kinetic equations for a ligand-gated channel to be solved analytically (see Methods). In a previous paper (Destexhe et al., 1994b) we demonstrated that pulse-based methods could provide reasonable models of postsynaptic responses and gave solutions for a first-order kinetic scheme. Here, we extend these results to three-state models (solutions given in Appendix A). We compared the behavior of two and three-state models to both more detailed models and to postsynaptic currents recorded using whole-cell voltage-clamp methods.

We began by simulating a detailed, six-state model for the AMPA/kainate receptor derived by Standley et al. (1993; Eq. 25), based on single channel recordings from locust muscle. Fig. $4 \mathrm{E}$ shows the time course of the AMPA/kainate PSC producted by the Standley model in conjunction with the detailed model of 

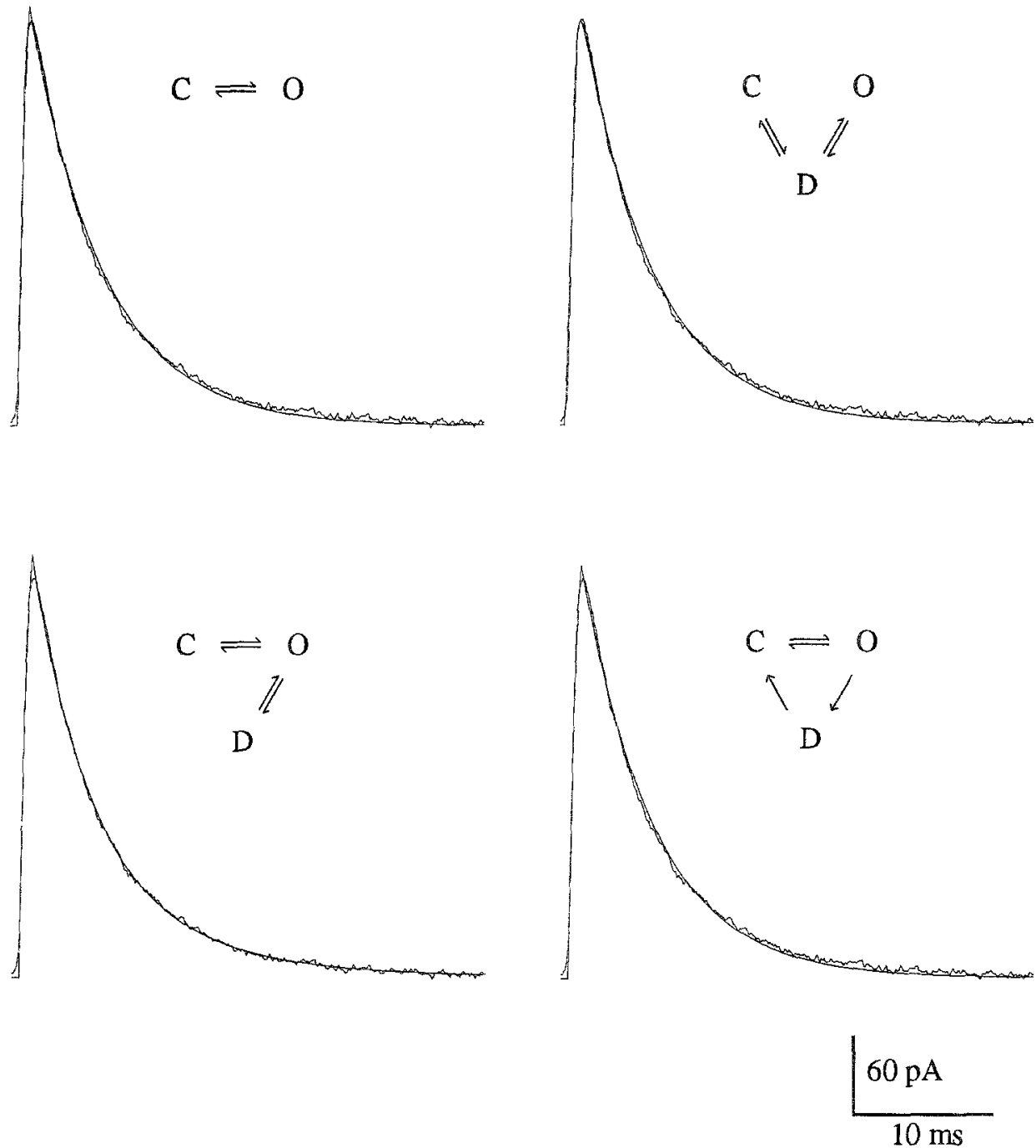

Fig. 6. Best fits for several simple kinetic schemes to averaged postsynaptic current mediated by AMPA/kainate receptors. The kinetic schemes were based on a $1 \mathrm{mM}$ rectangular pulse of transmitter lasting $1 \mathrm{~ms}$ using the parameters listed in Table 1. The kinetic equations were solved exactly, leading to an analytic expression for the postsynaptic current. The best fit obtained with each model (continuous traces) is compared with AMPA/kainate -receptor-mediated postsynaptic currents (noisy traces - negative upwards). Averaged recording of AMPA/kainate-receptor-mediated postsynaptic currents were obtained by whole-cell recordings at about $31^{\circ} \mathrm{C}$ in mossy fiber synapses in CA3 pyramidal cells (Xiang et al., 1992).

transmitter release described above. The postsynaptic response showed a fast time to peak of about $1 \mathrm{~ms}$ and a decay phase lasting 5-10 $\mathrm{ms}$, in agreement with Standley et al. (1993).

In experiments using a fast-perfusion technique, Colquhoun et al. (1992) and Hestrin (1992) found that $1 \mathrm{~ms}$ pulses of glutamate applied to patches containing AMPA/kainate receptor-gated channels produced responses that closely matched the time course of synaptic currents. We therefore had reason to believe that $1 \mathrm{~ms}$ pulses of transmitter applied to Markov models of AMPA/kainate channels could yield reasonable postsynaptic responses.

We found that two and three-state kinetic schemes with $1 \mathrm{~ms}$ transmitter pulses fit well the average AMPA/kainate PSC's obtained by whole-cell recordings (Fig. 6), in this case recorded from mossy-fiber synapses onto CA3 neurons in the hippocampal slice. Although the second-order model fit was better, the first-order model is reasonably accurate. Several com- 
Table 1. Elementary kinetic schemes and rate constants for transmitter-gated ion channels. Optimal values of the rate constants obtained by fitting elementary gating kinetic schemes to averaged recordings of synaptic currents for various transmitter-gated receptors (see Fig. 6). An analytic expression obtained for the time course of the current (see Appendix A) was fit to recordings using a simplex algorithm. First- and second-order kinetic schemes were used assuming a pulse of transmitter of $1 \mathrm{~ms}$ duration and $1 \mathrm{mM}$ amplitude. AMPA/kainate-mediated currents $\left(31^{\circ} \mathrm{C}\right)$ were provided by Z. Xiang and T. Brown; NMDA-mediated currents $\left(22-25^{\circ} \mathrm{C}\right)$ were provided by N.A. Hessler and R. Malinow; $\mathrm{GABA}_{A}$-mediated currents $\left(33-35^{\circ} \mathrm{C}\right)$ were provided from T. Otis and I. Mody. The symbols for the rate constants correspond to the kinetic scheme in Eq. (24).

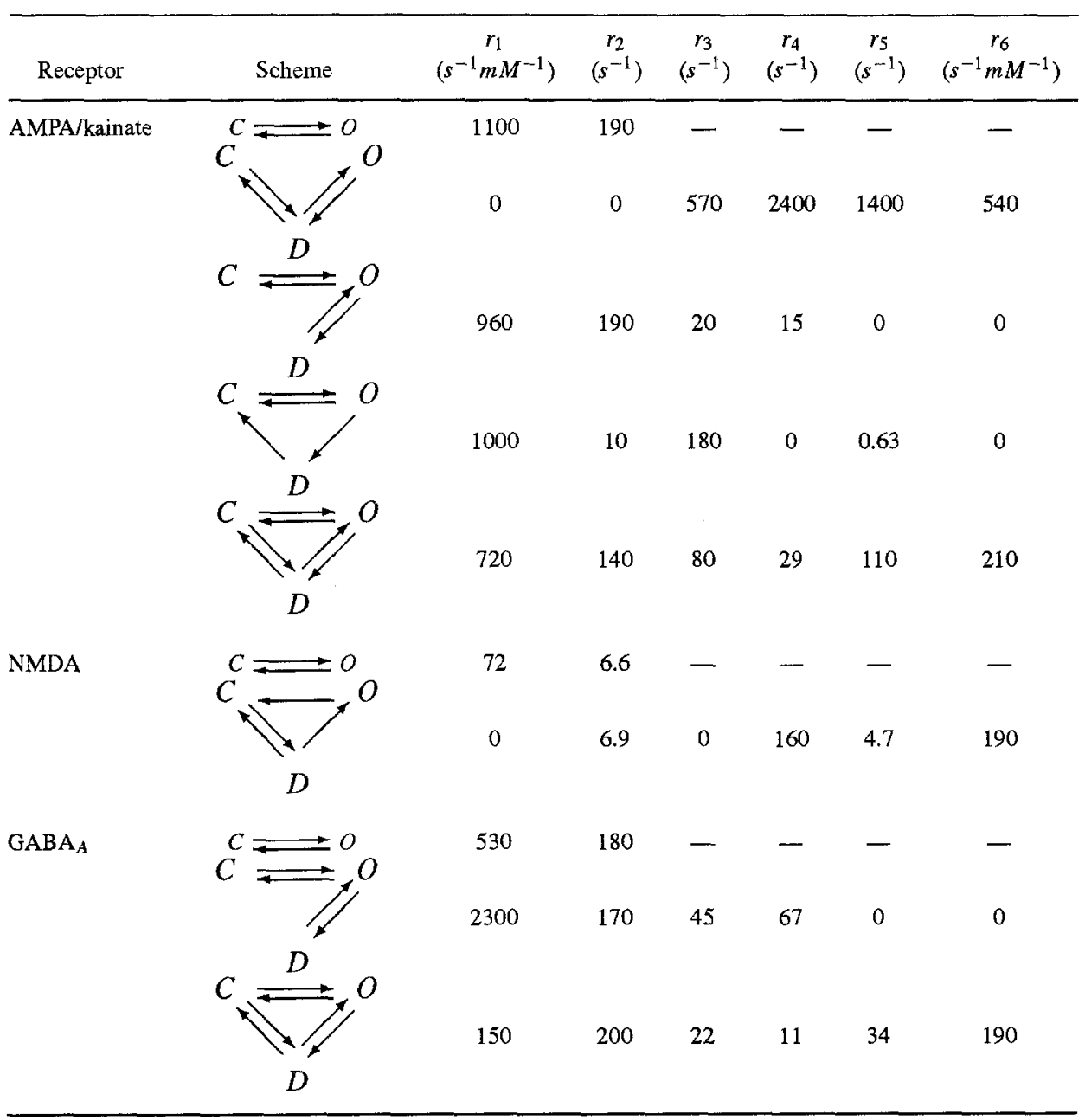

binations of the rate constants for different versions of the second-order model were found to provide equally good fits of the single averaged PSC (see Table 1).

The detailed release model and the six-state AMPA/kainate model were simulated and a series of presynaptic action potentials were elicited at a rate of approximately $20 \mathrm{~Hz}$ by injection of current (Fig. 7). Under these conditions there was a progressive desensitization of the response due to the increase of the fraction of desensitized channels (states $D_{1}$ and $D_{2}$ ). A similar behavior was also found with another model of the AMPA/kainate current (Raman and Trussell, 1992; not shown).

Again, the simple two and three-state kinetic schemes triggered by transmitter pulses were compared to the detailed model. The three-state model

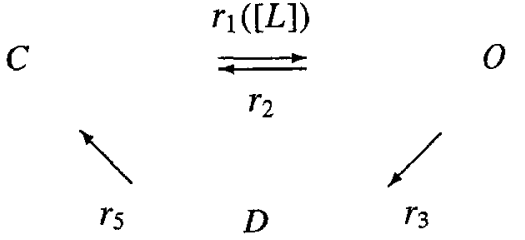


A Presynaptic voltage
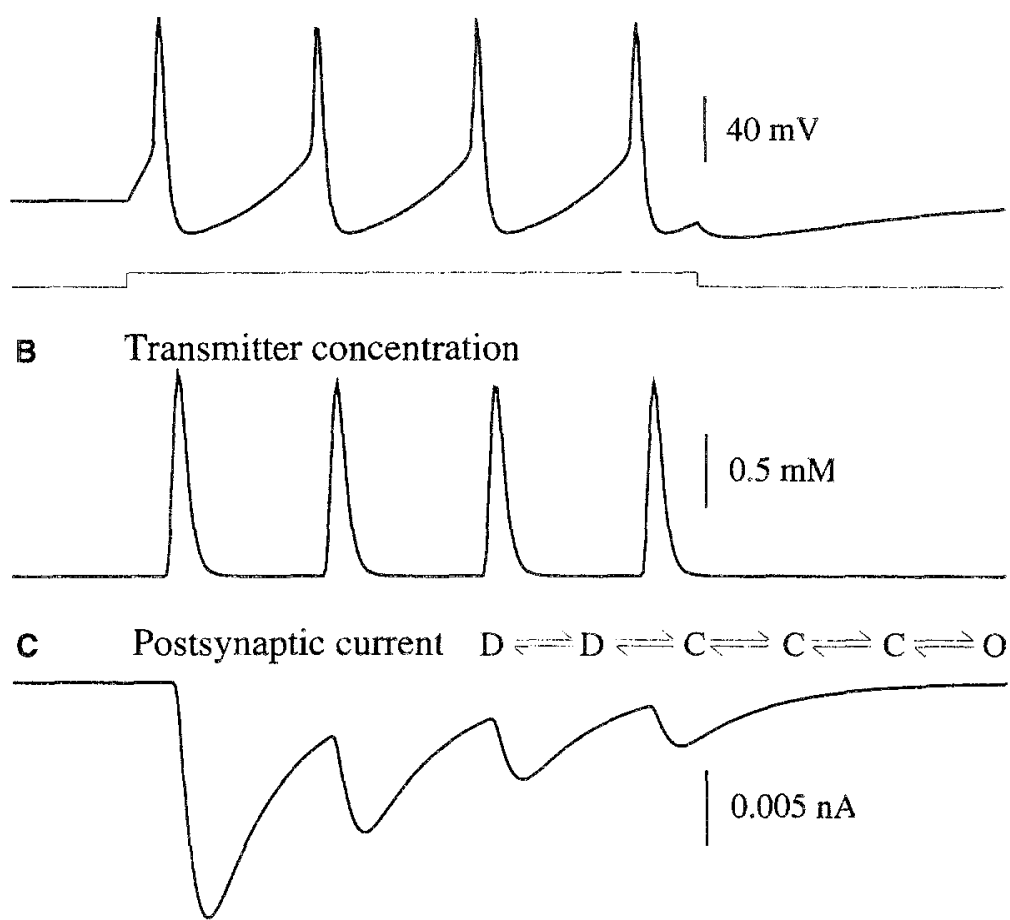

D

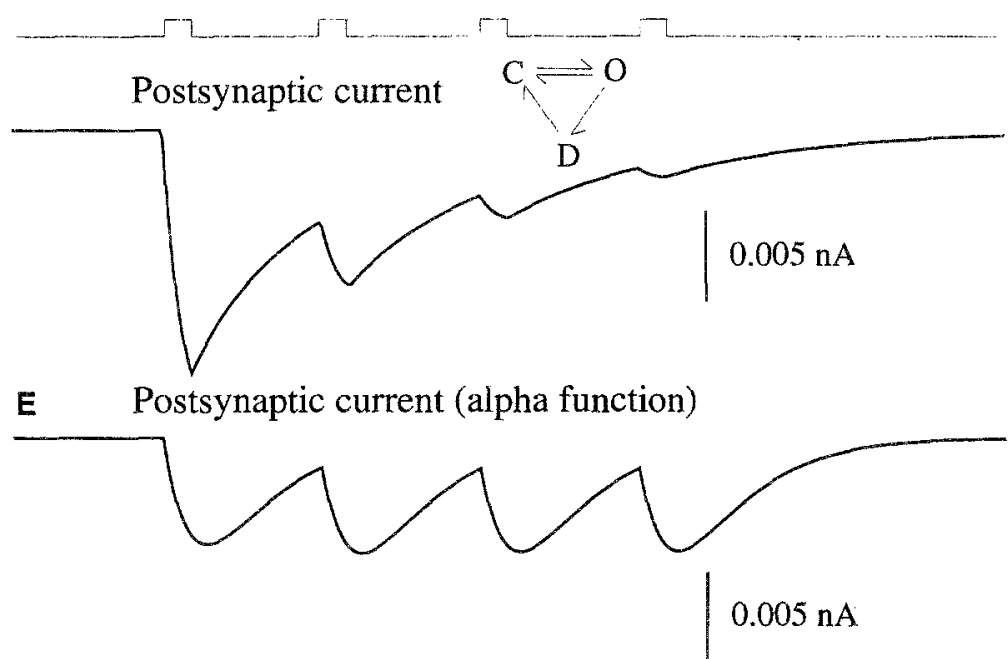

$10 \mathrm{~ms}$

Fig. 7. Comparison of models for AMPA/kainate receptors. A. Presynaptic train of four action potentials elicited by injection of a $20 \mathrm{~ms}$ current pulse of $0.1 n \mathrm{~A}$. B. Corresponding volley of glutamate release in the synaptic cleft obtained using the kinetic model of transmitter release. C. Postsynaptic current from AMPA/kainate receptors modeled by kinetic scheme in Eq. (25) (all parameters used in A-C were identical to those in Fig. 4). The progressive decrease of the amplitude of the successive excitatory postsynaptic currents is characteristic of the presence of desensitized states. D. Same simulation with AMPA/kainate receptors modeled by an elementary kinetic scheme (Eq. 38 with $r_{1}=1000 s^{-1} m M^{-1}, r_{2}=10 s^{-1}, r_{3}=180 s^{-1}, r_{4}=0, r_{5}=0.63 s^{-1}, r_{6}=0, \bar{g}=0.4 n S, E_{r e v}=0 m V$ ). For this case, the transmitter time course was approximated by a series of $1 \mathrm{mM}$ pulses lasting $1 \mathrm{~ms}$ (indicated above the current trace). E. Same simulation as in $\mathrm{D}$, but the postsynaptic current was modeled by a series of summated alpha functions $\left(t_{1}=1.6 m s, \bar{g}=0.1 \mathrm{nS}, E_{r e v}=0 \mathrm{mV}\right)$. In all cases the peak depolarization did not exceed $-60 \mathrm{mV}$. 
was found to be the best approximation for AMPA/kainate current. The time course of the PSCs in this simple model reproduced the progressive desensitizing responses (Fig. 7D) as well as the time course of the AMPA/kainate current observed in the more accurate model (25).

Figure 7 also illustrates the summation of postsynaptic currents obtained using alpha functions (Eq. 22). In this case (Fig. 7E), there was no desensitization, and the fit of alpha functions to the the time course of the AMPA/kainate PSC's was poor (the rise time was too slow compared to the decay time). The two-state model (Eq. 23) provided good fits of single PSC's (see Fig. 6), but was also unable to account for desensitization.

Similar procedures were followed for two other types of synaptic currents, namely those gated by the NMDA subtype of glutamate receptor and the $\mathrm{GABA}_{A}$ receptor. First- and second-order schemes were also fit to an averaged PSC obtained by wholecell recording (see Methods). The optimal values of the kinetic constants obtained from these fits are shown in Table 1.

\subsection{Second Messenger-Gated Ion Channels}

A variety of neurotransmitters lead to the opening or the closing of postsynaptic channels through a second messenger. For a large class of receptors, a G-protein subunit might directly gate an associated potassium channel (see reviews by North, 1989; Hille, 1992; Brown and Birnbaumer, 1992). Single channel recordings have demonstrated that a variety of potassium channels are gated by application of purified G-protein subunits (VanDongen et al., 1988). Various neurotransmitter receptors have been shown to act through this pathway and are summarized in Table 2. To demonstrate the scope of the kinetic formalism, we investigated the application of Markov kinetics to models of this class of slow or modulatory synaptic action. We followed the same general approach as for fast synapses, examining both detailed and simple kinetic models.

We considered a kinetic model for the gating of $K^{+}$channels through the G-protein subunit $G_{\alpha}$. The actions of the $G_{\beta \gamma}$ subunit, which include phosphorylation of other receptors (Clapham and Neer, 1993), were left for a future extension of the model. We made the following assumptions: (a) all receptors
Table 2. G-protein gating of neuronal $K^{+}$channels. Direct G protein-mediated activation of a potassium channel is a general mechanism of transmitter action in the central nervous system. The neurotransmitters listed here, acting through the corresponding receptor subtypes are likely to have a direct G-protein mediated pathway to the activation of $K^{+}$channels. Other pathways involving G-proteins have been found but are not listed here, and channels other than $\mathrm{K}^{+}$channels are regulated by G-proteins (see reviews by Neer and Clapham, 1988; Nicoll, 1988; North, 1989; Ross, 1989; Brown, 1990; Brown and Birnbaumer, 1990; Birnbaumer, 1992; Clapham and Neer, 1993).

\begin{tabular}{lcl}
\hline Transmitter & Receptor & \multicolumn{1}{c}{ Reference } \\
\hline GABA & GABA & Andrade et al., 1986 \\
Serotonin & $5 H T-1$ & Williams et al., 1988 \\
Acetylcholine & muscarinic $M 2$ & Sasaki and Sato, 1987 \\
Noradrenaline & $\alpha 2$ & Surprenant and North, 1988 \\
Dopamine & $D 2$ & Innis and Aghajanian, 1987 \\
Adenosine & $A 1$ & Trussell and Jackson, 1987 \\
Histamine & & Sasaki and Sato, 1987 \\
Opioids & $\delta, \mu$ & North et al., 1987 \\
Somatostatin & & Inoue et al., 1988 \\
\hline
\end{tabular}

considered (Table 2) share a common G-protein pathway with the same kinetics; (b) the differences between individual receptor actions are due to particular properties of the associated $K^{+}$channel; (c) the $K^{+}$channel is voltage-independent; (d) G-proteins obey conventional enzyme kinetics, ie the effects of the diffusion of G-proteins within the membrane are negligible (but see Lamb and Pugh, 1992).

G-protein gating of the $K^{+}$channel was modeled by the following kinetics scheme (see Fig. 1):

$$
\begin{aligned}
& L+R \underset{g_{2}}{\stackrel{g_{1}}{\rightleftharpoons}} R^{*} \\
& R^{*}+G \stackrel{g_{3}}{\underset{g_{4}}{\longrightarrow}} R G \stackrel{g_{5}}{\longrightarrow} R^{*}+G_{\alpha}^{*}+G_{\beta \gamma} \\
& G_{\alpha}^{*} \stackrel{k_{c a t}}{\longrightarrow} G_{\alpha} \\
& G_{\alpha}+G_{\beta \gamma} \stackrel{g_{6}}{\rightleftarrows} G
\end{aligned}
$$




$$
G_{\alpha}^{*}+C \rightleftharpoons \cdots \rightleftharpoons O
$$

The transmitter binds to receptor $(R)$ leading to an activated receptor $\left(R^{*}\right)$ with rate constants $g_{1}$ and $g_{2}$ (Eq. 39). $R^{*}$ then catalyzes the fission of the "resting" G-protein $(G)$ into activated $G_{\alpha}^{*}$ and $G_{\beta \gamma}$ subunits, with rate constants $g_{3}, g_{4}$ and $g_{5}$ (Eq. 40). The "activated" alpha subunit $G_{\alpha}^{*}$ is hydrolyzed into the "inactivated" form $G_{\alpha}$ (Eq. 41) which binds to $G_{\beta \gamma}$ to reconstitute $G$ (Eq. 42 ), with respective rate constants $k_{\text {cat }}, g_{6}$ and $g_{7}$. Finally, the $G_{\alpha}^{*}$ subunit acts as the ligand of a $K^{+}$channel according to Markov kinetic schemes (Eq. 43 and see Methods).

Here, $G$ represents the GDP-bound, "resting," form of the G-protein. In Eq. (40), GDP is released from $G$ and replaced by GTP, leading to a GTP-bound G-protein form that spontaneously beaks into the $G_{\beta \gamma}$ and the GTP-bound $G_{\alpha}^{*}$ subunit (Breitweiser and Szabo, 1988; Ross, 1989; Lopez, 1992). This complex process of G-protein activation by GDP/GTP exchange is represented implicitly in Eq. (40). The activated (GTP-bound) form $G_{\alpha}^{*}$ is then hydrolyzed into the inactive (GDP-bound) form $G_{\alpha}$ by GTPase (Eq. 41 ).

Three components are essential to the kinetics of G-protein linked responses (see review by Ross, 1989): (a) The release of GDP from $G$, corresponding to rate $g_{5}$, is the rate-limiting step in the activation process. The release of GDP has been shown to be proportional to the concentration of agonist in cardiac myocytes in vivo (Breitweiser and Szabo, 1988). (b) The decay of activated protein is dominated by the hydrolysis reaction ( $k_{c a t}$ in Eq. 41). The lower bound on this rate has been estimated to be on the order of 2-3 $s^{-1}$ (Breitweiser and Szabo, 1988). (c) The response is highly amplified. For one molecule of activated receptor $\left(R^{*}\right)$, about 20 to 1000 molecules of G-protein are activated. G-proteins are also present in much higher concentration than receptors in the membrane and a significant fraction of the total locallyavailable pool of G-proteins is activated during the stimulation of the pathway (see Ross, 1989).

Figure 8 shows an implementation of the G-protein kinetic scheme, with the time course of the different protein species during the release of transmitter. The case of the GABA $B$-mediated current was chosen here because its kinetics have been characterized (Otis et al., 1993) and there is strong evidence that direct $\mathrm{G}$-protein binding mediates the gating process
(Andrade et al., 1986; Thalmann, 1988; Brown and Birnbaumer, 1990). In the model illustrated in Fig. 8, a very brief increase in transmitter concentration gave rise to a much longer duration intracellular response, with rise and decay times comparable to that estimated by pharmacological manipulations and recordings in vivo (Breitweiser and Szabo, 1988; Szabo and Otero, 1989). In this simulation, the release was obtained according to the release model of the preceding section, and the $K^{+}$channel was gated by $G_{\alpha}^{*}$ using a three-state Markov scheme (Eq. 38). The rate constants were fit to the time course of $\mathrm{GABA}_{B}$ currents measured by whole-cell voltage clamp in dentate granule cells (Otis et al., 1993).

Figure 9 shows another type of $\mathrm{G}$ protein-linked response, that mediated by $\alpha 2$ noradrenergic receptors. In this example, the time course of the PSC was quite different than the $K^{+}$current activated by $\mathrm{GABA}_{B}$ receptors (see North, 1989). We assumed that the time course of the intracellular G-protein pathway was identical, but that the kinetics of the $K^{+}$ channel activated by $\alpha 2$ noradrenergic receptors differed. Figure 9A-C shows the slow hyperpolarizing current and voltage in response to a single presynaptic spike. The longer lasting effect of a train of presynaptic spikes is shown in Fig. 9D-E. In this case, tonic but low frequency presynaptic firing, generated a sustained hyperpolarization in the postsynaptic cell.

Simplified models for G-protein-gated channels were also considered. We assumed that the concentration of the $G_{\alpha}^{*}$ subunit could be approximated by a pulse a few tens of $m s$ long (see Fig. 8). With such a pulse, two and three-state Markov models were fit (see Methods) to the averaged GABA $_{B}$ PSC's (Otis et al., 1993; Fig. 10). The two-state model agreed with the overall time course of the current. Threestate schemes were able to fit either the rise or the decay of the $\mathrm{GABA}_{B}$ PSC, but not both. Ultimately, the four-state scheme

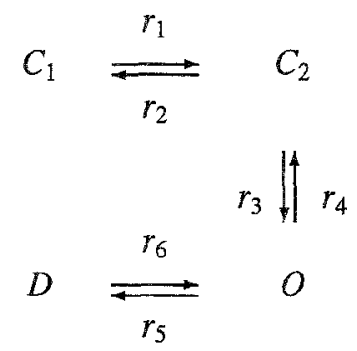


A

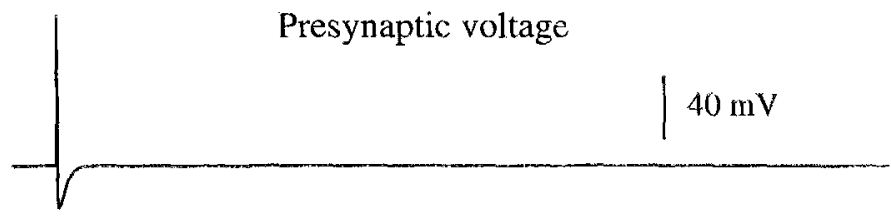

B

Transmitter concentration

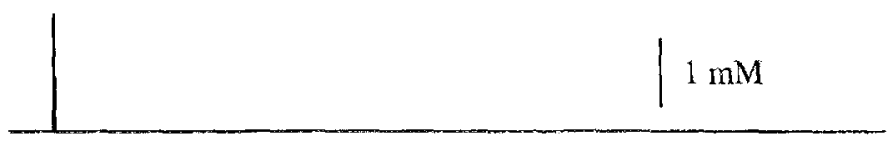

c

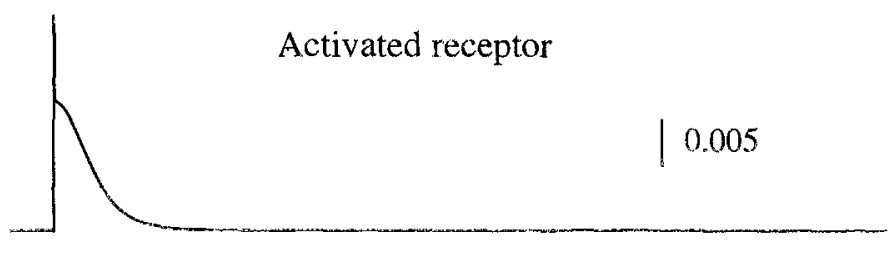

D

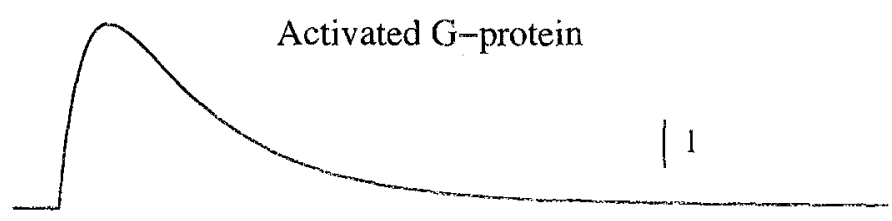

E

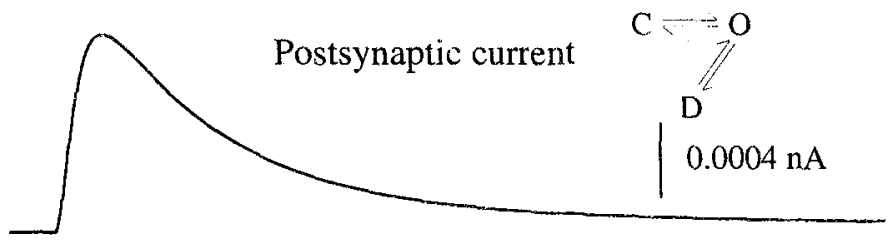

$\mathbf{F}$

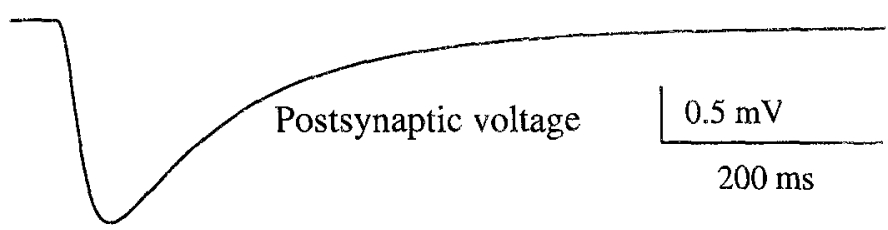

Fig. 8. Kinetic models of presynaptic release and binding to $\mathrm{GABA}_{B}$ receptors, a second-messenger-gated ion channel. A. Presynaptic action potential elicited by current injection. B. Time course of the transmitter, GABA, in the synaptic cleft using the same model for transmitter release as in Fig. 4A-D. C. Activated form $R^{*}$ of the GABA $B$ receptors after binding with GABA (the concentration was renormalized to $\left.1 ; g_{1}=400 s^{-1} m M^{-1}, g_{2}=500 s^{-1}\right)$. D. $G_{\alpha}^{*}$, the activated G-protein subunit, catalyzed by $R^{*}$ The concentration of $G_{\alpha}^{*}$ was renormalized and was 10 times in excess with respect to other proteins $\left(g_{3}=2000 s^{-1}, g_{4}=100 s^{-1}, g_{5}=1000 s^{-1}, g_{6}=1000 s^{-1}\right.$, $g_{7}=0.1 s^{-1}, k_{c a t}=10 s^{-1}$ ). The activated G-protein subunit $G_{\alpha}^{*}$ gates a postsynaptic ion channel according to Markov kinetics. E. Postsynaptic current produced by the gating of a $K^{+}$channel by the activated G-protein subunit $G_{\alpha}^{*}$ according to the kinetic scheme in Eq. (24) $\left(r_{1}=18 s^{-1} m M^{-1}, r_{2}=50 s^{-1}, r_{3}=10 s^{-1}, r_{4}=2 s^{-1}, r_{5}=0, r_{6}=0, \bar{g}_{G A B A}=0.1 n S\right)$. F. Inhibitory postsynaptic potential (leakage conductance was $0.2 \mathrm{mS} / \mathrm{cm}^{2}$ and leakage reversal potential was $-70 \mathrm{mV}$ ). 

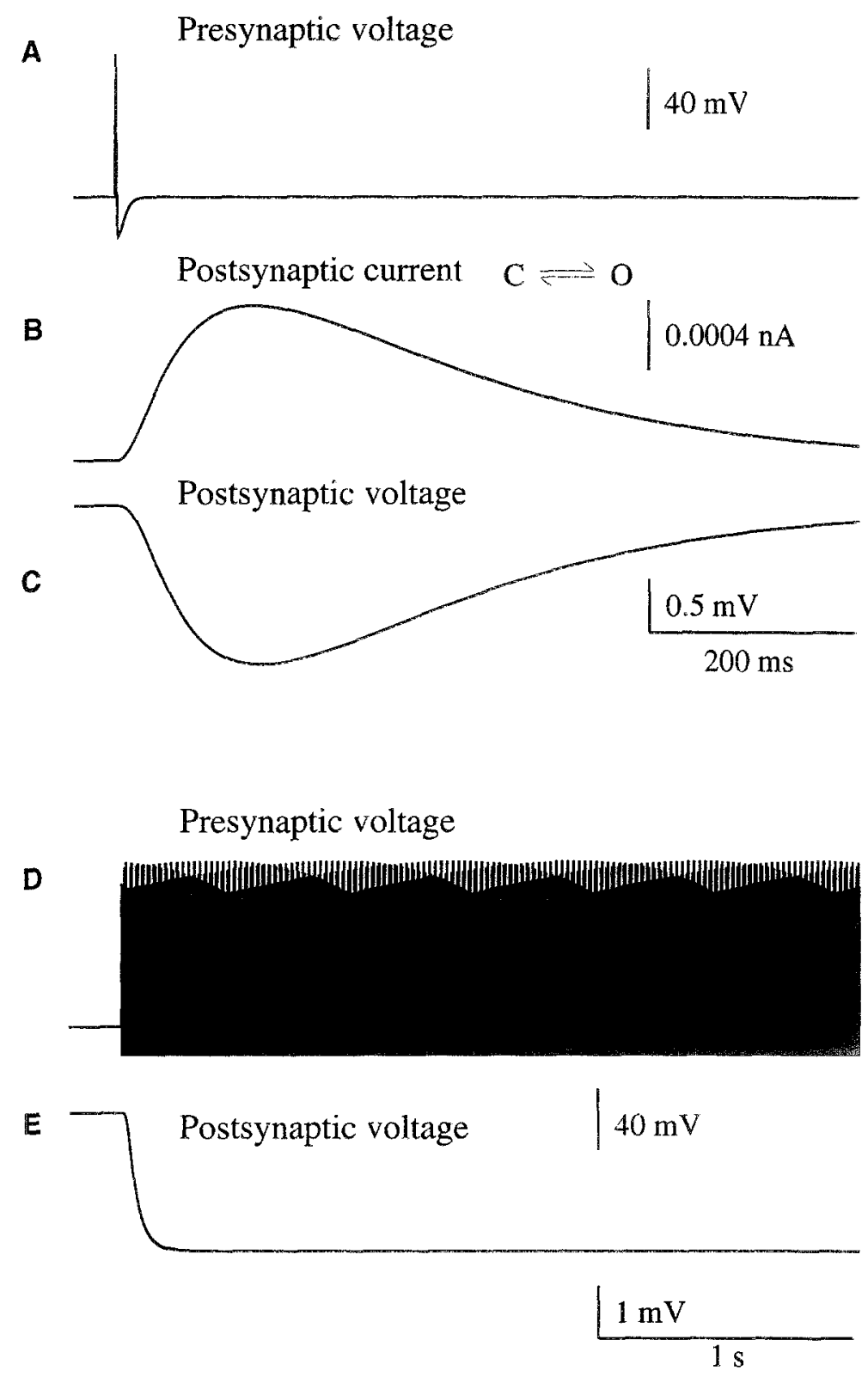

Fig. 9. Kinetic model of neuromodulation via noradrenergic $\alpha 2$ receptors. A. Presynaptic spike obtained by current injection. B. G-proteinmediated opening of $\mathrm{K}^{+}$channels according to first-order kinetics $\left(r_{1}=0.002, r_{2}=0.007\right.$; other parameters as in Fig. 8). C. Slow inhibitory postsynaptic potential following the presynaptic spike. D. Tonic presynaptic activity obtained by injection of a sustained current of $0.1 \mathrm{nA}$. E. Presynaptic drive elicited a sustained hyperpolarization of the postsynaptic membrane. 

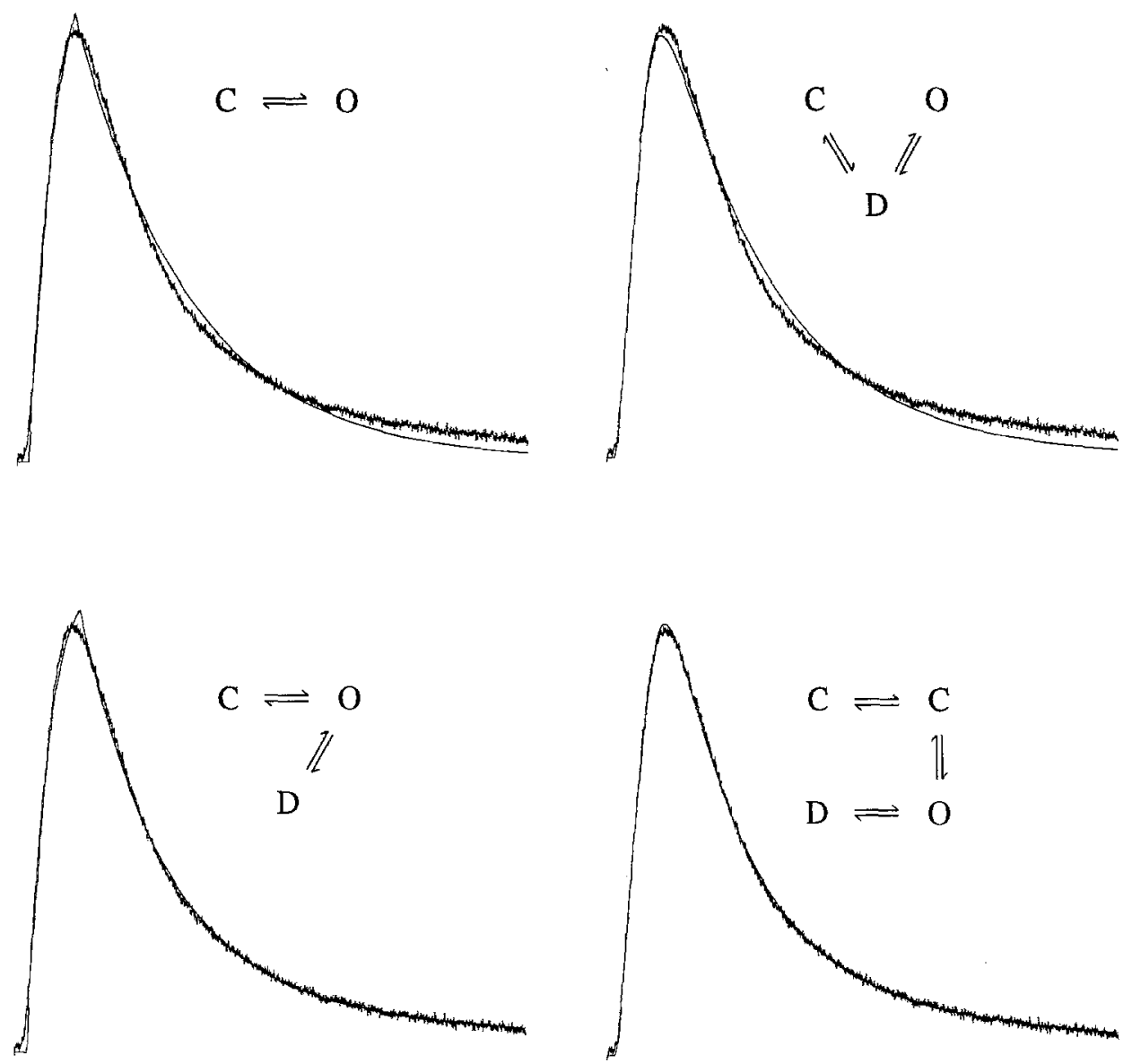

$10 \mathrm{pA}$

$200 \mathrm{~ms}$

Fig. 10. Best fits for several simple kinetic schemes to averaged postsynaptic current mediated by GABA $B$ receptors. $1 \mu M$ pulses of activated G-protein lasting 60 to $100 \mathrm{~ms}$ were used in the kinetic model (parameters as listed in Table 1). The best fit obtained with each model (continuous trace) is compared to experimental measurements of $\mathrm{GABA}_{B}$-mediated postsynaptic currents (noisy traces). Averaged recordings of $\mathrm{GABA}_{B}$-mediated postsynaptic currents were obtained by whole-cell recordings in dentate granule cells at $33-35^{\circ} \mathrm{C}(\mathrm{Otis}$ et al., 1993).

was found to be the minimal model which combined the kinetic features necessary to fit both the rise and decay phases, though the number of free parameters for this scheme was no more than for the three-state model.

Table 3 gives estimated values of rate constants for Markov models of a number of G-protein mediated conductances, including as $\mathrm{GABA}_{B}, 5 H T-1$ (serotonin), muscarinic $M 2$ (acetylcholine), $\alpha 2$ (norepinephrine), D2 (dopamine), histamine, $\delta$ and $\mu$ (opioids) and somatostatin.

\section{Discussion}

In this paper, we have explored models for a variety of cellular processes, including the gating of channels by voltage, the calcium-dependent enzyme cascade implicated in transmitter release, the gating of channels by neurotransmitter, and the G-protein cascade underlying the action of a number of neuromodulators on a class of potassium channels. We have illustrated that the same formalism can be used to describe all these mechanisms and generated simplified models 
Table 3. Elementary kinetic schemes and rate constants for G-protein gated potassium channels. Optimal values of the rate constants obtained by fitting elementary gating kinetic schemes to direct G protein-gated synaptic currents. The same procedure was used as described in Table 1 for first- and second- order kinetic schemes, assuming a pulse of G-protein of $1 \mu M$ amplitude. For higher-order kinetics, direct fit of the kinetic model to the data was performed (see Methods). The rate constants of G-protein mediated currents were estimated from published recordings (Breitweiser and Szabo, 1988; North 1989; see also references in Table 2); the parameters of GABA $B$ receptor-mediated currents were estimated from recordings provided by T. Otis and I. Mody (see Fig. 10). The duration of the pulse is given in the last column. The symbols for the rate constants correspond to the kinetic scheme in Eq. (24). $\left(^{*}\right)$ : units of $s^{-1}$.

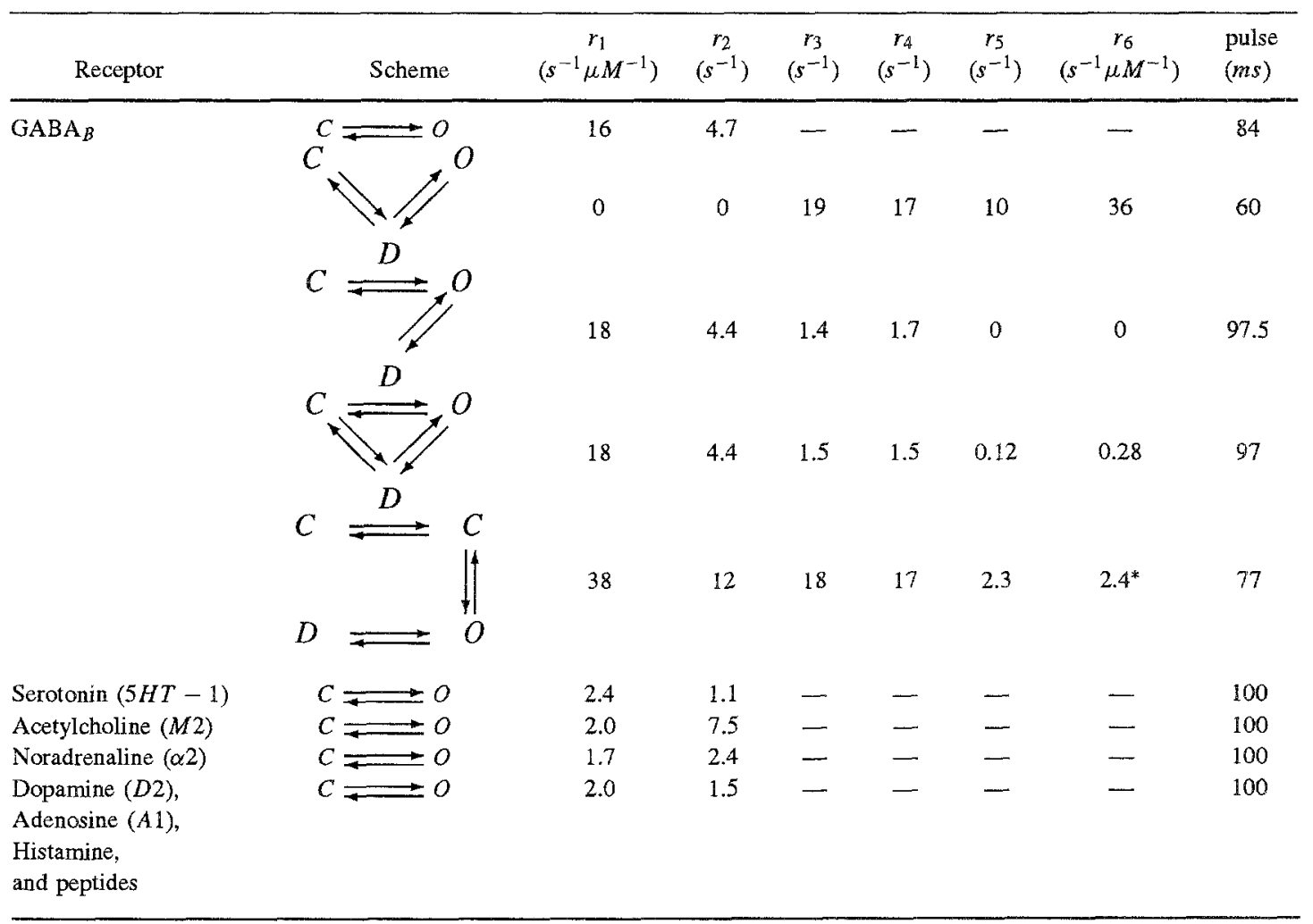

that are computationally efficient. Here we will discuss the assumptions as well as the advantages and drawbacks of this approach and indicate directions for further applications.

\subsection{Assumptions of Markov Kinetics}

Finite-state models of ion channels rely in general on the assumption that the configuration of a channel protein in the membrane can be operationally grouped into a set of distinct states separated by large energy barriers (Hille, 1992). Because the flux of ions though single channels can be directly measured, it has been possible to observe directly the predicted rapid and stochastic transitions between conducting or open and non-conducting closed states (Neher, 1992). Channels can be treated as finite-state Markov systems if one further assumption is made, namely that the probability of state transitions is dependent only of the present state occupied.

The assumption that channels function through a succession of conformational changes does not lead inevitably to a finite-state Markov description, and alternative treatments have been given. Diffusional (Millhauser et al., 1988) or continuum gating models (Levitt, 1989), are Markovian but posit an infinite number of states. Fractal (Liebovitch and Sullivan, 1987), or deterministically chaotic (Liebovitch and Toth, 1991) models assume a finite number of states, but allow time-dependent transition rates. Differentiation between discrete multistate Markov models and any of these alternatives hinges on high timeresolution studies of channel openings. Analysis of single-channel openings and closings has been consis- 
tent with the suitability of finite-state Markov models (McManus et al., 1988; Sansom et al., 1989).

In this paper, we have concentrated exclusively on macroscopic ionic currents rather than their discrete and stochastic counterparts at the microscopic level. In order to arrive at equations for such macroscopic kinetics it was necessary to assume that in all cases the number of channels contributing to an ionic current would be sufficiently large to wash out variability from single channel events. This assumption will break down in cases in which extremely low channel density or minute membrane areas are considered, having possible consequences on electrical signalling (Clay and DeFelice, 1983; Strassberg and DeFelice, 1993). Thus channel noise might be relevant at small structures such as the dendritic spines at which most central excitatory synapses are made (Strassberg and DeFelice, 1993; also see below).

\subsection{Models of Voltage-Gated Channels}

The model of the fast sodium current and the delayed rectifier potassium current introduced by Hodgkin and Huxley (1952) was remarkably forward looking. The general framework, based on four independent gating particles, is echoed in the multiple subunit channel structure revealed by molecular techniques. The Hodgkin-Huxley description not only accounts very well for the conductances of the squid giant axon, but has been widely applied, with very minor alterations, to describe nearly the entire scope of voltage-dependent currents (see e.g. Yamada et al., 1989; Borg-Graham, 1991; Lytton and Sejnowski, 1991). Nevertheless, there is considerable physiological and molecular evidence that channel gating is organized differently from what is suggested by Hodgkin-Huxley kinetics (reviewed in Armstrong, 1992). Activation and inactivation are clearly coupled in a multi-state sequence rather than operating independently.

The Markov kinetic formalism advocated here was adopted by many of the recent biophysical models of the kinetics of voltage-gated channels, such as sodium channels (Aldrich et al., 1983; Chabala, 1984; Horn and Vandenberg, 1984; Keller et al., 1986; Aldrich and Stevens, 1987; Clay, 1989 Vandenberg and Bezanilla, 1991), potassium channels (Labarca et al., 1985; Hoshi and Aldrich, 1988; Perozo and
Bezanilla, 1990), calcium channels (Chen and Hess, 1990), chloride channels (Labarca et al., 1980), and voltage-dependent gap junctions (Harris et al., 1981; Chanson et al., 1993). The kinetic formalism is more general than the Hodgkin-Huxley framework, including it as a special case, and is more flexible and extensible. Within the kinetic framework it is possible to include more states, making the model more biophysically accurate, or fewer states, providing a spectrum of models from which to chose depending on the nature of the idealization. A model that focussed on the network level, for example, might choose a simpler version that captured the primary properties of the channel. We have provided examples of how this can be accomplished for the sodium channel and have shown that the approximations of the minimal model is very reasonable. A three-state irreversible loop model was found by Bush and Sejnowski (1991) to reproduce well a number of voltage-dependent channels in Purkinje cells. In the other direction, improvements in our biophysical knowledge of channels can be seamlessly folded into models without having to enter an entirely new framework.

We used two-state (first-order) and three state (second-order) kinetic schemes as simple models of voltage-dependent currents. These basic schemes have been analyzed in general by Kienker (1989). We considered rates given by a saturating, sigmoidal function of voltage rather than a simple exponential as is commonly assumed in Markov models (e.g. Chabala, 1984; Vandenberg and Bezanilla, 1991; Perozo and Bezanilla, 1990; Harris et al., 1981). The increase in complexity of the voltage-dependence allows a model with fewer states to reproduce more complex voltage-dependent channel behavior (Keller et al., 1986; Clay, 1989; Chen and Hess, 1990). We have found that the two-state model captures the features of the delayed rectifier potassium current, a noninactivating current, and a three-state model is the minimal representation of the fast sodium current, an inactivating current.

We suggest that this approach can be extended by considering these models as prototypes of simplified models of most of voltage-dependent currents. One strategy would be to begin, as we did, with detailed models, such as those derived from singlechannel recordings, and take steps to reduce the number of states required. Alternatively, the availability of 
analytic solutions for time constants and equilibrium values (Appendix A) would facilitate fitting of two- and three-state models directly to voltage-clamp recordings.

\subsection{Models of Transmitter-Gated Channels}

Our understanding of the action of neurotransmitters began with studies of endplate currents at the neuromuscular junction (Katz, 1966). Early quantitative descriptions of these currents made use of simple kinetic models (e.g. Katz, 1966; Magleby and Stevens, 1972). The advent of single-channel recording saw the application of Markov models to the behavior of individual ligand-gated channels (Colquhoun and Hawkes, 1977; Sakmann and Neher, 1983). As improved physiological, pharmacological, and molecular techniques have revealed additional complexities, the complexity of the models has increased, but a new formalism has not been necessary. Currently, rigorous quantitative accounts of synaptic channels may easily dictate a Markov scheme of well over a half dozen states (Raman and Trussel, 1992; Standley et al., 1993) without even taking into account allosteric effects.

In the present study, we have shown that when efficiency and speed are concerns, simple two- and three-state kinetic schemes can capture both the time course of the postsynaptic currents and the interaction of successive events. This contrasts with the standard alpha function approach, which, while simple and inexpensive to compute, has no natural provision for summation or saturation of consecutive events. We have given general analytic solutions for the two- and three-state kinetic schemes (Appendix A) that can be applied under conditions of constant agonist concentration.

A useful approximation that allows the analytical solution to be exploited is to model the time-course of transmitter in the synaptic cleft as a discrete pulse (Staubli et al., 1992; Destexhe et al., 1994b). In this way, the transmitter is always constant at either high or low concentration (during or between pulses, respectively). The computation involved is comparable to that for a properly-implemented alpha function (see Srinivasan and Chiel, 1993), with no more than one exponential calculation required for the two-state model or two for the three-state model.
We have generally assumed that the time-course of postsynaptic responses are dominated by the receptor kinetics rather than the availability of transmitter (e.g. Lester et al., 1990). There is reason to believe that the approximation of a $1 \mathrm{~ms}$ pulse is an acceptable representation. Experimental measurements of the time-course of glutamate at a hippocampal synapse have indicated a near instantaneous rise in concentration followed by a decay with a time constant of around $1 m s$ (Clements et al., 1992). Furthermore, one millisecond pulses of glutamate applied to outside-out patches of hippocampal and cortical neurons produce AMPA/kainate receptor-mediated currents with time-courses very close to that recorded in the intact synapse (Colquhoun et al., 1992; Hestrin, 1992).

Analytic solutions based on kinetic models have been obtained by considering the time-course of transmitter as a delta-function (e.g. Perkel et al., 1981; Holmes and Levy, 1992). We considered pulses of transmitter, which produced a model that was still analytically tractable, but somewhat more flexible than the delta-function. Most importantly, by increasing the length of the pulse, we were able to mimic the extended time course of second-messenger activation in order to reproduce the behavior of G-protein-linked potassium channels.

Kinetic equations involving concentrations, fractions of channels, and rate constants rely on ensemble averages over the activity of a large populations of channels or other proteins. These assumptions break down when a single synapse is considered. First, the release of transmitter-containing vesicles upon spike invasion is a probabilistic process (Katz, 1966). Whereas our kinetic release model gives reliably the same amount of transmitter following each action potential, probabilities of transmitter release of less than 0.1 have been measured at central synapses (Rosenmund et al., 1993; Hessler et al., 1993). One could take into account this stochasticity by replacing the kinetic equations for release with a pulse-based model in which the pulse was triggered following a presynaptic action potential conditional on a random variable.

A second problem in the application of kinetic equations to individual synapses arises when the relatively small number of postsynaptic receptors at a release site is taken into account. The liberation of a quantum of neurotransmitter at central synapses 
is currently thought to open only about 5-50 postsynaptic receptors (Edwards et al., 1990; Hestrin, 1992; Traynelis et al., 1993). Consequently, individual synaptic currents may have significant variability due to probabilistic channel openings (see e.g. Silver et al., 1992). In situations in which this variability is of interest, stochastic rather than kinetic models would be appropriate. A model incorporating Markov kinetics could in such cases be reimplemented using Monte-Carlo methods in order to simulate the behavior of individual channels rather than population averages (see e.g. Wathey et al., 1979; Bartol et al., 1991).

\subsection{Models of Second Messenger-Gated Channels}

Generally, G protein-linked receptors mediate a wide range of physiological responses, many of which involve the modulation of ion channels. We have presented a relatively simple model for a class of potassium channels gated directly by G-proteins. In contrast to voltage-dependent channels or transmittergated channels, relatively little single-channel data is available for second messenger-gated channels. This is primarily due to technical obstacles. The gating of these channels requires a collection of proteins and chemical reactants located either intracellularly or in the membrane, and therefore recordings from isolated patches of membrane require the presence of these chemical and protein species in the solutions (see VanDongen et al., 1988).

A model of another $G$ protein-mediated pathway was proposed recently for photoreceptor activation (Lamb and Pugh, 1992). However in this case the G-protein does not directly activate the ion channels, but acts via a second messenger, namely, cyclic GMP. Another difference is that the kinetics in phototransduction are substantially faster than those of neuromodulatory pathways (Ross et al., 1992). Nevertheless, it could be shown that the kinetics of phototransduction could be accounted for in a quantitative fashion by using a detailed model based on standard chemical kinetics (Lamb and Pugh, 1992).

Another example of a G-protein pathway involving second messengers is the regulation of the hyperpolarization-activated current, $I_{h}$. This current is the common target of a number of neuromodulators, such as histamine, adenosine, noradrenaline and sero- tonin (reviewed in McCormick, 1992). In this case, several different receptors catalyze the formation of the same second messenger, cyclic AMP, which regulates the voltage-dependence of $I_{h}$ (see DiFrancesco and Matteo, 1994). It would make sense to model such an interplay of modulatory actions using a kinetic approach similar to the one we have presented here.

Finally, many G-protein pathways are known to play a role in biochemical processes beyond ion channel modulation. An important example is the involvement of G-proteins in synaptic vesicle cycling (Hess et al., 1993). As many $G$ protein-linked receptors, such as the $\mathrm{GABA}_{B}$ receptor, are also found presynaptically, a range of interactions leading to the regulation of the release process are possible. A natural extension of the models presented here would be to combine the synaptic release and G-protein cascades to examine these interactions.

\subsection{Methods of Simplification}

We provided several examples of how reduced kinetic descriptions can reproduce the dynamics of ion channel behavior. There are more systematic ways to identify such reduced models. For example, Kienker (1989) showed how to establish the equivalence of different models and to reduce the number of their parameters, by a procedure similar to that used in classical mechanics. A particular transformation can be shown to reorganize the states and rates of the system, leading in some cases to uncoupled variables, thereby reducing the number of independent variables of the system. Such a procedure can be used to obtain a reduced model which is formally equivalent to the initial model. However, a similar method could also be applied to reduce the system using various approximations for obtaining uncoupled variables (Kepler et al., 1992). A significant advantage of following such a procedure is that it automatically produces the rate constants for the simplified model.

Other ways to simplify the Hodgkin-Huxley equations have been proposed (e.g. Fitzhugh, 1961; Krinskii and Kokoz, 1973; Hindmarsh and Rose, 1982; Rinzel, 1985; Keppler et al.,1992). A common simplification is to assume that fast reactions are always close to their equilibrium values. This is generally applicable to multi-state kinetic schemes if some rates 
are orders of magnitude faster than others. For example, if the binding step in the activation of a ligandactivated receptor is sufficiently rapid compared to subsequent conformational changes leading to channel opening, then the initial two states may cease to be kinetically distinguishable and can be effectively collapsed into one (Colquhoun and Hawkes, 1977). As another example, the interaction of magnesium ions with the NMDA receptor has been simplified by assuming (a) the kinetics of the magnesium block are fast compared to the kinetics of the channel, so that the amount of block can be considered at steadystate; and (b) the block acts directly at the channel pore without infuencing the intrinsic gating of the channel (Jahr and Stevens, 1990b; and see Methods).

The assumption that the transmitter concentration in the synaptic cleft is given by a function of the presynaptic voltage leads to a simplified description of synaptic interaction. A relatively steep sigmoid relationship emerged, roughly corresponding to the activation function of the presynaptic calcium current, raised to the fourth power. A similar sigmoid function was used by Wang and Rinzel (1992) to represent synaptic interactions in a kinetics-based model that uses graded potentials rather than fast spikes.

\subsection{Application to Interacting Neurons}

An important theoretical application of the kinetic description presented here is to the analysis of neural interactions. A general kinetic approach allows an entire network of neurons to be described by autonomous equations, even if individual cells contain voltagedependent currents or if they interact via secondmessenger pathways. The coupling between pre- and post-synaptic cells can be accomplished either by kinetic models, such as the presynaptic release mechanism we described, or more simply through functions which approximate the release process, such as Eq. (37). The set of equations describing a network of neurons might be extremely complex, but there are several possible applications.

The full set of equations describing the network could constitute the starting point of a theoretical treatment, with an aim to eventually derive greatly simplified representations. The simplified equations obtained could be the basis for abstract neural network models, perhaps yielding novel algorithms for cellular or synaptic function. This "bottom-up" strategy contrasts with the more common tactic of neural network models in which the properties of single cells are simplified in ad hoc manner.

In addition, the set of autonomous equations describing the network could be used to study the global dynamics of neural populations. A considerable amount of experimental data is available from measurements of the average activity of populations of brain cells: recordings of electroencephalogram, 10cal field potentials, magnetoencephalograms, optical recordings, magnetic resonance images, etc. It would be interesting to attempt to establish a relationship between such global measurements and dynarnics at the molecular level, which might be done through an approach analogous to statistical mechanics.

A final important application is toward understanding the role of specific intrinsic properties of neurons in the organization of the collective dynamics of neural populations. In central neurons, the presence of intrinsic currents can confer to the cell extremely complex responsiveness (Llinás, 1988). As these complex intrinsic properties are often combined with connectivity involving many different types of receptors, the dynamics of interconnected populations of such neurons becomes extremely difficult to comprehend intuitively (see Destexhe et al., 1993b, 1994a). In addition, the activity of various transmitters can modulate the intrinsic properties of the cells (McCormick, 1992; Lopez and Brown, 1992), thereby modifying collective properties of the network. For example, diffuse ascending neurotransmitter systems control the shift of the thalamocortical system from fast processing during arousal to slow wave activity during sleep (Steriade and McCarley, 1990; Steriade et al., 1993). We expect that the kinetic formalism will prove useful in modeling the influences of these neuromodulatory systems.

\subsection{Molecular and Biochemical Applications}

Although we have mainly addressed the utility of employing simple Markov models for the description of voltage-dependent and synaptic currents, it is also important to stress the benefits of constructing ion channel models in a language that is compatible with molecular and biochemical descriptions. 
Significant progress has been made in recent years in relating the function of ion channels to specific elements of their molecular structure (reviewed in Unwin, 1989; Catterall, 1992; Andersen and Koeppe, 1992; Armstrong, 1992; Jan and Jan, 1992; Sakmann, 1992). Markov models, because they are subject to direct physical interpretation, are well-suited to incorporating the insights from such a level of analysis. In this respect, the Markov kinetic approach is significantly more general than other approaches, such as the Hodgkin-Huxley model for voltage-gated channels or the alpha-function model for synaptic responses. As the physiological significance of protein regulation is further elucidated, a compatible functional description will become increasingly valuable.

In the present paper, we have given only a few examples of the wide range of biological phenomena that can be addressed by models that consider ion channels in a molecular and biochemical context as well as an electrical one. Computational models have already begun to address a number of these instances. As a prominent example, the gating of channels by second-messengers is a ubiquitous and critical phenomenon (Partidge and Swandulla, 1988; Latorre et al., 1989; Toro and Stefani, 1991) and calciumdependent gating of potassium channels has already been widely utilized in neural models (e.g. Yamada et al., 1989; Lytton and Sejnowski, 1991; Destexhe et al., 1993b).

Relatively direct interactions between secondmessengers, G-proteins and receptors mark only the initial stages in the integration of biochemical and physiological models that can be accomplished in a kinetic framework such as the one we have outlined. Protein phosphorylation, one of the most important mechanisms in the short and long-term regulation of neural function (reviewed by Walaas and Greengard, 1991) represents an important target for quantitative description. The detailed potassium channel model that was illustrated here was formulated by Perozzo and Bezanilla (1989) to provide a biophysical account of the effect of phosphorylation on the channel's voltage-gating properties, indicating the effectiveness of a Markov formalism in addressing these problems.

The regulation of synaptic efficacy has been perhaps the most intensely studied arena of such biochemical interactions, and in this domain, both simple and detailed kinetic models (Lisman, 1989; Skene,
1990; Holmes and Levy, 1990; Zador et al., 1990; Ambros-Ingerson and Lynch, 1993; Migliore and Ayala, 1993) have already begun to provide insight. Activity-dependent regulation of channel properties has likewise begun to be investigated in such a fashion (Bell, 1992; LeMasson et al., 1993). In large part, the intricate web of second-messengers, protein phosphorylation systems, and the deeper machinery of signal transduction and gene regulation still await integration into computational models of neural activity. The kinetic framework provides a natural way of integrating electrophysiology with cellular biochemistry, in which ion channels are considered as a special and important class of enzymes rather than as a completely distinct subject.

\section{Acknowledgments}

This research was supported by the Howard Hughes Medical Institue, the Office of Naval Research and the National Institutes of Mental Health. Z.F.M. is a Howard Hughes Medical Institute Predoctoral Fellow. We thank Drs. T. Brown, N.A. Hessler, R. Malinow, I. Mody, T. Otis, and Z. Xiang for use of their data, and $P$. Kienker for useful comments on the manuscript.

NEURON programs that simulate the models of this article are available on request, or by anonymous ftp to salk.edu in /pub/alain.

\section{Appendix A Analytic Expressions of the Current for Elementary Kinetic Schemes}

\subsection{First-Order Kinetics}

A general scheme for a ligand- or voltage-gated channel obeying first-order kinetics is:

$$
C \stackrel{r_{1}(V,[L])}{\underset{r_{2}(V)}{\rightleftarrows}} O
$$

where the symbols are as defined before for Eq. (23). The current resulting from a population of such channels is obtained by Eq. (28).

Assume that kinetic rates are constant, or subject to a step transition. In the case of ligand-gated post- 
synaptic currents, this corresponds to a pulse of transmitter. In the case of voltage-dependent currents, this corresponds to a voltage clamp, and the solution given below is the time course of the current following a voltage jump.

The kinetic equation is:

$$
\frac{d y}{d t}=r_{1}(V,[L])(1-y)-r_{2}(V) y
$$

where $y$ is the fraction of open channels.

Let the system be in an initial state $y=y_{0}$ with $V=V_{0}$ and $[L]=[L]_{0}$ for $t<t_{0}$. At $t=t_{0}$, a change in $[L]$ and $V$ occurs such as $V=V_{1}$ and $[L]=[L]_{1}$ for $t>t_{0}$. It is then straightforward to solve Eq. (46), giving:

$y\left(t-t_{0}\right)=y_{\infty}+K_{1} \exp \left[-\left(t-t_{0}\right) / \tau_{1}\right]$

where

$$
\begin{aligned}
& K_{1}=y_{0}-y_{\infty} \\
& y_{\infty}=\frac{r_{1}\left(V_{1},[L]_{1}\right)}{r_{1}\left(V_{1},[L]_{1}\right)+r_{2}\left(V_{1}\right)} \\
& \tau_{1}=\frac{1}{r_{1}\left(V_{1},[L]_{1}\right)+r_{2}\left(V_{1}\right)} .
\end{aligned}
$$

\subsection{Second-Order Kinetics}

A general kinetic scheme for ion channels gated by second-order kinetics is:

C

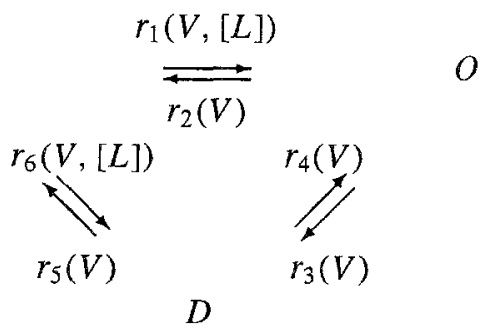

where the symbols are as defined before for Eq. (24).

The corresponding kinetic equations are:

$$
\begin{aligned}
\frac{d y}{d t}= & r_{1}(V,[L])(1-y-z)- \\
& {\left[r_{2}(V)+r_{3}(V)\right] y+r_{4}(V) z }
\end{aligned}
$$

$$
\begin{aligned}
\frac{d z}{d t}= & r_{6}(V,[L])(1-y-z)- \\
& {\left[r_{4}(V)+r_{5}(V)\right] z+r_{3}(V) y }
\end{aligned}
$$

where $y$ is the fraction of open channels $(O)$ and $z$ is the fraction of desensitized channels $(D)$.

Let the system be in an initial state $y=y_{0}$ and $z=z_{0}$ with $V=V_{0}$ and $[L]=[L]_{0}$ for $t<t_{0}$. At $t=t_{0}$, a change in $[L]$ and $V$ occurs such as $V=V_{1}$ and $[L]=[L]_{1}$ for $t>t_{0}$. The general solution of the system is:

$$
\begin{aligned}
y\left(t-t_{0}\right)= & y_{\infty}+K_{1} \exp \left(-\left(t-t_{0}\right) / \tau_{1}\right) \\
& +K_{2} \exp \left(-\left(t-t_{0}\right) / \tau_{2}\right) \\
z\left(t-t_{0}\right)= & z_{\infty}+K_{3} \exp \left(-\left(t-t_{0}\right) / \tau_{1}\right) \\
& +K_{4} \exp \left(-\left(t-t_{0}\right) / \tau_{2}\right)
\end{aligned}
$$

where

$$
\begin{aligned}
& K_{1}=\frac{\left(y_{0}-y_{\infty}\right)\left(a+1 / \tau_{2}\right)+b\left(z_{0}-z_{\infty}\right)}{1 / \tau_{2}-1 / \tau_{1}} \\
& K_{2}=\left(y_{0}-y_{\infty}\right)-K_{1} \\
& K_{3}=K_{1} \frac{-a-1 / \tau_{1}}{b} \\
& K_{4}=K_{2} \frac{-a-1 / \tau_{2}}{b} \\
& y_{\infty}=\frac{b r_{6}\left(V_{1},[L]_{1}\right)-d r_{1}\left(V_{1},[L]_{1}\right)}{a d-b c} \\
& z_{\infty}=\frac{c r_{1}\left(V_{1},[L]_{1}\right)-a r_{6}\left(V_{1},[L]_{1}\right)}{a d-b c}
\end{aligned}
$$

and the coefficients are $a=-r_{1}\left(V_{1},[L]_{1}\right)-r_{2}\left(V_{1}\right)-$ $r_{3}\left(V_{1}\right), b=-r_{1}\left(V_{1},[L]_{1}\right)+r_{4}\left(V_{1}\right), c=r_{3}\left(V_{1}\right)-$ $r_{6}\left(V_{1},[L]_{1}\right)$ and $d=-r_{4}\left(V_{1}\right)-r_{5}\left(V_{1}\right)-r_{6}\left(V_{1},[L]_{1}\right)$. The time constants $\tau_{1}$ and $\tau_{2}$ are given by:

$\tau_{1,2}^{-1}=-\frac{a+d}{2} \pm \frac{1}{2} \sqrt{(a-d)^{2}+4 b c}$

If $\tau_{1}=\tau_{2}$, the system is described by a single exponential with the following values:

$$
\begin{aligned}
K_{1}= & {\left[\left(y_{0}-y_{\infty}\right)\left(a+1 / \tau_{1}\right)+b\left(z_{0}-z_{\infty}\right)\right] } \\
& \left(t-t_{0}\right)+\left(y_{0}-y_{\infty}\right)
\end{aligned}
$$




$$
\begin{aligned}
K_{3}= & {\left[\frac{\left(y_{0}-y_{\infty}\right)\left(a+1 / \tau_{1}\right)+b\left(z_{0}-z_{\infty}\right)}{b}\right] } \\
& {\left[1-\left(a+1 / \tau_{1}\right)\left(t-t_{0}\right)\right]+} \\
& \left(y_{0}-y_{\infty}\right) \frac{-a-1 / \tau_{1}}{b}
\end{aligned}
$$

and with $K_{2}=K_{4}=0$.

\section{Appendix B Kinetic Scheme and Derivation of the Alpha Function}

Consider the following second-order gating scheme:

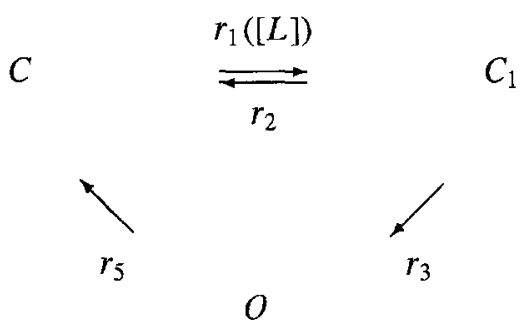

where $C$ and $C_{1}$ are the closed forms of the receptor, $O$ is the open (conducting) form, and $r_{1} \ldots r_{5}$ are voltage-independent rate constants.

This scheme can give rise to an alpha function only if two approximations can be made:

(a) The transmitter concentration ([L]) occurs as a Dirac delta function $\delta\left(t-t_{0}\right)$ (i.e. a pulse of infinitely large amplitude and infinitely short duration). For a release event occurring at time $t=t_{0}$ one writes:

$$
r_{1}([L])=r_{1} \delta\left(t-t_{0}\right) .
$$

(b) The form $C$ is always considered to be in excess compared to $C_{1}$ and $O$. This would be the case if very few receptors bind transmitter, so that nearly all receptor molecules remain in form $C$. The fraction of channels in $C$ is therefore considered as constant and always remains $\sim 1$.

Making these assumptions, one obtains the following set of kinetic equations:

$$
\begin{aligned}
& \frac{d x}{d t}=r_{1} \delta\left(t-t_{0}\right)-\left(r_{2}+r_{3}\right) x \\
& \frac{d y}{d t}=r_{3} x-r_{5} y,
\end{aligned}
$$

where $x$ and $y$ represent the fraction of receptors in the forms $C_{1}$ and $O$ respectively.

These equations can be solved by Laplace transform, giving the following general doubleexponential solution for $y$ :

$$
\begin{aligned}
& y\left(t-t_{0}\right)= \\
& r_{1} r_{3} \frac{\exp \left[-\left(t-t_{0}\right) / \tau_{1}\right]-\exp \left[-\left(t-t_{0}\right) / \tau_{2}\right]}{1 / \tau 2-1 / \tau_{1}},
\end{aligned}
$$

where $\tau_{1}=1 /\left(r_{2}+r_{3}\right)$ and $\tau_{2}=1 / r_{5}$.

In the limit of $\tau_{2} \rightarrow \tau_{1}$, or $r_{5} \rightarrow\left(r_{2}+r_{3}\right)$, this reduces to

$$
y\left(t-t_{0}\right)=r_{1} r_{3}\left(t-t_{0}\right) \exp \left[-\left(t-t_{0}\right) / \tau_{1}\right],
$$

which is a form equivalent to the alpha function introduced by Rall (1967).

It is important to mention that this kinetic interpretation of the alpha function only holds for $y \ll 1$, in order to remain consistent with condition (b) above.

\section{References}

Aldrich RW, Corey DP and Stevens CF (1983) A reinterpretation of mammalian sodium channel gating based on single channel recording. Nature 306: 436-441.

Aldrich RW and Stevens CF (1983) Inactivation of open and closed sodium channels determined separately. Cold Spring Harbor Symp. Quant. Biol. 48: 147-153.

Aldrich RW and Stevens CF (1987) Voltage-dependent gating of single sodium channels from mammalian neuroblastoma cells. J. Neurosci. 7: 418-431.

Ambros-Ingerson J and Lynch G (1993) Channel gating kinetics and synaptic efficacy: a hypothesis for expression of long-term potentiation. Proc. Natl. Acad. Sci. USA 90: 7903-7909.

Andersen O and Koeppe RE II (1992) Molecular determinants of channel function. Physiol. Rev. 72: S89-S158.

Andrade R, Malenka RC and Nicoll RA (1986) A G protein couples serotonin and $\mathrm{GABA}_{B}$ receptors to the same channels in hippocampus. Science (Wash.) 234: 1261-1265.

Armstrong CM (1969) Inactivation of the potassium conductance and related phenomena caused by quaternary ammonium ion injection in squid axons. J. Gen. Physiol. 54: 553-575.

Armstrong CM (1981) Sodium channels and gating currents. Physiol. Rev. 62: 644-683.

Armstrong CM (1992) Voltage-dependent ion channels and their gating. Phyiol. Rev. 72: S5-S13.

Augustine GJ and Charlton MP (1986) Calcium dependence of presynaptic calcium current and post-synaptic response at the squid giant synapse. J. Physiol. (London) 381:619-640.

Ball FG and Sansom MS (1989) Ion-channel gating mechanisms: model identification and parameter estimation from single channel recordings. Proc. Roy. Soc. Lond. Ser. B 236: 385-416.

Bartol TM Jr, Land BR, Salpeter EE and Salpeter MM (1991) Monte Carlo simulation of miniature endplate current generation 
in the vertebrate neuromuscular junction. Biophys. J. 59: 12901307.

Bell AJ (1992) Self-organisation in real neurons: anti-Hebb in 'channel space'? In: Moody JE, Hanson SJ and Lippmann RP, eds. Advances in Neural Information Processing Systems 4, Morgan-Kaufmann, San Mateo, CA. pp. 59-66.

Bezanilla F (1985) Gating of sodium and potassium channels. J. Membr. Biol. 88: 97-111.

Birnbaumer L (1992) Receptor-to-effector signaling through G proteins: roles for beta gamma dimers as well as alpha subunits. Cell 71: 1069-1072.

Blaustein MP (1988) Calcium transport and buffering in neurons. Trends Neurosci. 11: 438-443.

Borg-Graham LJ (1991) Modeling the nonlinear conductances of excitable membranes. In: Wheal $\mathrm{H}$ and Chad J, eds. Cellular and Molecular Neurobiology: A Practical Approach. Oxford University Press, New York. pp. 247-275.

Breitwieser GE and Szabo G (1988) Mechanism of muscarinic receptor-induced $K^{+}$channel activation as revealed by hydrolysis-resistant GTP analogues. J. Gen. Physiol. 91: 469493.

Bush P and Sejnowski TJ (1991) Simulations of a reconstructed cerebellar Purkinje cell based on simplified channel kinetics. Neural Computation 3: 321-332.

Brown DA (1990) G-proteins and potassium currents in neurons. Annu. Rev. Physiol. 52: 215-242.

Brown AM and Birnbaumer L (1990) Ionic channels and their regulation by $\mathrm{G}$ protein subunits. Annu. Rev. Physiol. 52: 197213.

Catterall WA (1992) Cellular and molecular biology of voltagegated sodium channels. Physiol. Rev. 72: S15-48.

Chabala LD (1984) The kinetics of recovery and development of potassium channel inactivation in perfused squid giant axons. J. Physiol. (Lond.) 356: 193-220.

Chanson M, Chandross KJ, Rook MB, Kessler JA, Spray DC (1993) Gating characteristics of a steeply voltage-dependent gap junctional channel in rat Schwann cells. J. Gen. Physiol. 102: 925-946.

Chen C and Hess P (1990) Mechanisms of gating of T-type calcium channels. J. Gen. Physiol. 96: 603-630.

Clapham, DE and Neer EJ (1993) New roles for G-protein $\beta \gamma$ dimers in transmembrane signalling. Nature 365: 403-406.

Clay JR (1989) Slow inactivation and reactivation of the potassium channel in squid axons. Biophys. J. 55: 407-414.

Clay JR and DeFelice LJ (1983) Relationship between membrane excitability and single channel open-close kinetics. Biophys. J. 42: 151-157.

Clements JD, Lester RAJ, Tong J, Jahr C, and Westbrook GL (1992) The time course of glutamate in the synaptic cleft. Science (Wash.) 258: 1498-1501.

Clements JD and Westbrook GL (1991) Activation kinetics reveal the number of glutamate and glycine binding sites on the NMDA receptor. Neuron 7: 605-613.

Colquhoun D and Hawkes AG (1977) Relaxation and fluctuations of membrane currents that flow through drug-operated channels. Proc. Roy. Soc. Lond. Ser. B 199: 231-262.

Colquhoun D and Hawkes AG (1981) On the stochastic properties of single ion channels. Proc. Roy. Soc. Lond. Ser. B 211: 205235.

Colquhoun D, Jonas P and Sakmann B (1992) Action of brief pulses of glutamate on $A M P A /$ kainate receptors in patches from different neurons of rat hippocampal slices. J. Physiol. (London) 458, 261-287.

Destexhe A, Babloyantz A and Sejnowski TJ (1993a) Ionic mechanisms for intrinsic slow oscillations in thalamic relay neurons. Biophys. J. 65: 1538-1552.

Destexhe A, Contreras C, Sejnowski TJ and Steriade M (1994a) A model of spindle thythmicity in the isolated reticular thalamus. J. Neurophysiol., 72:803-818.

Destexhe A, Mainen Z and Sejnowski TJ (1994b) An efficient method for computing synaptic conductances based on a kinetic model of receptor binding. Neural Computation 6: 14-18.

Destexhe A, McCormick DA and Sejnowski TJ (1993b) A model of 8-10 Hz spindling in interconnected thalamic relay and reticularis neurons. Biophys. J. 65: 2474-2478.

Difrancesco D and Matteo M (1994) Modulation of single hyperpolarization-activated channels $\left(i_{f}\right)$ by cAMP in the rabbit sino-atrial node. J. Physiol. (London) 474: 473-482.

Edwards FA, Konnerth A and Sakmann B (1990) Quantal analysis of inhibitory synaptic transmission in the dentate gyrus of rat hippocampal slices: a patch-clamp study. J. Physiol. (London) 430: 213-249.

Fidzhugh $R$ (1961) Impulses and physiological states in models of nerve membrane. Biophys. J. 1: 445-466.

Fitzhugh R (1965) A kinetic model of the conductance changes in nerve membrane. J. Cell. Comp. Physiol. 66: 111-118.

Goldman L and Hahin R (1979) Sodium conductance kinetics. Solution of the general, linear three-state model. Cell Biophys. 1: $345-354$.

Hagiwara $N$ and Irisawa $H$ (1989) Modulation by intracellular $\mathrm{Ca}^{2+}$ of the hyperpolarization-activated inward current in rabbit single sino-atrial node cells. J. Physiol. (London) 409: 121-141.

Harris AL, Spray DC and Bennett MVL (1981) Kinetic properties of a voltage-dependent junctional conductance. J. Gen. Physiol. 77: 95-117.

Hess SD, Doroshenko PA and Augustine GJ (1993) A functional role for GTP-binding proteins in synaptic vesicle cycling. Science (Wash.) 259: 1169-1172.

Hessler NA, Shirke AM and Malinow R (1993) The probability of transmitter release at a mammalian central synapse. Nature 366 : $569-572$.

Hestrin S (1992) Activation and desensitization of glutamateactivated channels mediating fast excitatory synaptic currents in the visual cortex. Neuron 9:991-999.

Hille B (1992) Ionic Channels of Excitable Membranes. Sinauer Associates INC, Sunderland, MA.

Hindmarsh JL and Rose RM (1982) A model of the nerve impulse using two first-order differential equations. Nature 296: 162-164.

Hines M (1989) A program for simulation of nerve equations with branching geometries. Int. J. Biomed. Comput. 24: 55-68.

Hines M (1993) NEURON-A program for simulation of nerve equations. In: Eeckman, FH, ed. Neural Systems: Analysis and Modeling Kluwer Academic Publishers, Boston, MA. pp. 127136.

Hodgkin AL and Huxley AF (1952) A quantitative description of membrane current and its application to conduction and excitation in nerve. J. Physiol. (London) 117: 500-544.

Holmes WR and Levy WB (1990) Insights into associative longterm potentiation from computational models of NMDA receptormediate calcium influx and intracellular calcium concentration changes. J. Neurophysiol. 63: 1148-1168. 
Horn $\mathrm{R}$ and Lange $\mathrm{K}$ (1983) Estimating kinetic constants from single channel data. Biophys. J. 43: 207-223.

Horn RJ, Patlak J and Stevens CF (1981) Sodium channels need not open before they inactivate. Nature 291: 426-427.

Horn R and Vandenberg CA (1984) Statistical properties of single sodium channels. J. Gen. Physiol. 84: 505-535.

Innis RB and Aghajanian GK (1987) Pertussis toxin blocks autoreceptor-mediated inhibition of dopaminergic neurons in rat substantia nigra. Brain Res. 411: 139-143.

Inoue M, Nakajima S and Nakajima Y (1988) Somatostatin induces an inward rectification in rat locus coeruleus neurones through a pertussis toxin-sensitive mechanism. J. Physiol. (London) 407 : 177-198.

Jahr CE and Stevens CF (1990a) A quantitative description of NMDA receptor-channel kinetic behavior. J. Neurosci. 10: 18301837.

Jahr CE and Stevens CF (1990b) Voltage dependence of NMDAactivated macroscopic conductances predicted by single-channel kinetics. J. Neurosci. 10:3178-3182.

Jan LY and Jan YN (1992) Structural elements involved in specific $K^{+}$channel functions. Annu. Rev. Physiol. 54: 537-555.

Johnson FH, Eyring $\mathrm{H}$ and Stover BJ (1974) The theory of rate processes in biology and medicine, New York: John Wiley and Sons.

Katz B (1966) Nerve, Muscle and Synapse. New York: McGraw Hill Book Co.

Keller BU, Hartshorne RP, Talvenheimo JA, Catterall WA and Montal M (1986) Sodium channels in planar lipid bilayers. Channel gating kinetics of purified sodium channels modified by batrachotoxin. J. Gen. Physiol. 88:1-23.

Kepler TB, Abbott LF and Marder E (1992) Reduction of conductance-based neuron models. Biol. Cybernetics 66: 381387.

Kienker P (1989) Equivalence of aggregated Markov models of ion-channel gating. Proc. Roy. Soc. Lond. Ser. B 236: 269-309.

Koch C and Segev I (Editors) (1989) Methods in Neuronal Modeling. Cambridge, MA: MIT Press.

Kohn MC (1989) Computer modeling at the National Biomedical Simulation Resource. Computers and Mathematics with Applications 18: 919-924.

Krinskii VI and Kokoz YM (1973) Analysis of the equations of excitable membranes - 1. Reduction of the HodgkinHuxley equations to a second-order system. Biofizika 18: 506511.

Labarca P, Coronado R and Miller C (1980) Thermodynamic and kinetic studies of the gating behavior of a $\mathrm{Kt}+$-selective channel from the sarcoplasmic reticulum membrane. J. Gen. Physiol. 76: 397-24.

Labarca P, Rice JA, Fredkin DR and Montal M (1985) Kinetic analysis of channel gating. Application to the cholinergic receptor channel and the chloride channel from Torpedo Californica. Biophys. J. 47: 469-478.

Lamb TD and Pugh EN (1992) A quantitative account of the activation steps involved in phototransduction in amphibian photoreceptors. J. Physiol. (London) 449: 719-758.

LeMasson G, Marder E and Abbott LF (1993) Activity-dependent regulation of conductances in model neurons. Science (Wash.) 259: 1915-1917.

Latorre R, Oberhauser A, Labarca P and Alvarez O (1989) Varieties of calcium-activated potassium channels. Annu. Rev. Physiol. 51: 385-99.
Lester RA and Jahr CE (1992). NMDA channel behavior depends on agonist affinity. J. Neurosci. 12: 635-643.

Levitt DG (1989) Continuum model of voltage-dependent gating. Biophys. J. 55: 489-498.

Liebovitch LS and Sullivan JM (1987) Fractal analysis of a voltagedependent potassium channel from cultured mouse hippocampal neurons. Biophys. J. 52: 979-988.

Lisman JE (1989) A mechanism for the Hebb and the anti-Hebb processes underlying learning and memory. Proc. Natl. Acad. Sci. USA 86: 9574.

Llinás RR (1988) The intrinsic electrophysiological properties of mammalian neurons: a new insight into CNS function. Science (Wash.) 242: 1654-1664.

Llinás RR, Steinberg IZ and Walton K (1981) Relationship between presynaptic calcium current and postsynaptic potential in squid giant synapse. Biophys. J. 33: 323-351.

Lopez HS (1992) Kinetics of G protein-mediated modulation of the potassium M-current in bullfrog sympathetic neurons. Neuron 8: 725-736.

Lopez HS and Brown AM (1992) Neuromodulation. Curr. Opin. Neurobiol. 2: 317-322.

Lytton WW and Sejnowski TJ (1991) Simulations of cortical pyramidal neurons synchronized by inhibitory interneurons. J. Neurophysiol. 66: 1059-1079.

MacDonald RL and Twyman RE (1992) Kinetic properties and regulation of $\mathrm{GABA}_{A}$ receptor channels. Ion Channels 3: 315343.

Magleby KL and Stevens CF (1972) A quantitative description of end-plate currents. J. Physiol. (London) 223: 173-197.

Marom, S and Abbott LF (1994) Modeling state-dependent inactivation of membrane currents. Biophys. J. 67:515-520.

Mayer ML, Westbrook GL and Guthrie PB (1984) Voltagedependent block by $\mathrm{Mg}^{2+}$ of NMDA responses in spine cord neurones. Nature 309: 261-263.

McCormick DA (1992) Neurotransmitter actions in the thalamus and cerebral cortex and their role in neuromodulation of thalamocortical activity. Progr. Neurobiol. 39: 337-388.

McCormick DA and Huguenard JR (1992) A model of the electrophysiological properties of thalamocortical relay neurons. J. Neurophysiol. 68: 1384-1400.

McCormick DA and Williamson A (1991) Modulation of neuronal firing mode in cat and guinea pig LGNd by histamine: possible cellular mechanisms of histaminergic control of arousal. J. Neurosci. 11: 3188-3199.

McManus OB, Weiss DS, Spivak CE, Blatz AL and Magleby KL (1988) Fractal models are inadequate for the kinetics of four different ion channels Biophys. J. 54: 859-870.

Migliore M and Ayala GF (1993) A kinetic model of short- and long-term potentiation. Neural Computation 5: 636-647.

Mihara S and Nishi S (1989) Muscarinic excitation and inhibition of neurons in the submucous plexus of the guinea-pig caecum. Neurosci. 31: 247-257.

Millhauser GL, Saltpeter EE and Oswald RE (1988) Diffusion models of ion-channel gating and the origin of power-law distributions from single-channel recording. Proc. Natl. Acad. Sci. USA 85: 1503-1507.

Monod J, Changeux JP and Jacob F (1963) Allosteric proteins and cellular control systems. J. Mol. Biol. 6: 306-329.

Neer EJ and Clapham DE (1988) Roles of G protein subunits in transmembrane signalling. Nature 333: 129-134. 
Neher E (1992) Ion channels for communication between and within cells. Science (Wash.) 256: 498-502.

Neher E and Sakmann B (1976) Single-channel currents recorded from membrane of denervated muscle frog fibers. Nature 260 : 799-802.

Neher E and Stevens CF (1979) Voltage-driven conformational changes in intrinsic membrane proteins. In: FO Schmitt and FG Worden, eds. The neurosciences. Fourth study program, MIT Press, Cambridge, MA, pp. 623-629.

Nicoll RA (1988) The coupling of neurotransmitter receptors to ion channels in the brain. Science (Wash.) 241: 545-551.

North RA (1989) Drug receptors and the inhibition of nerve cells. Brit. J. Pharmacol. 98: 13-28.

North RA, Williams JT, Surprenant A and Christie, MJ (1987) $\mathrm{Mu}$ and delta receptors belong to a family of receptors that are coupled to potassium channels. Proc. Natl. Acad. Sci. USA 84: $5487-5491$.

Nowak L, Bregestovski P, Ascher P, Herbet A and Prochiantz A (1984) Magnesium gates glutamate-activated channels in mouse central neurones. Nature 307: 462-465.

Otis TS, Dekoninck Y and Mody I (1993) Characterization of synaptically elicited $\mathrm{GABA}_{B}$ responses using patch-clamp recordings in rat hippocampal slices. J. Physiol. (London) 463: 391-407.

Otis TS and Mody I (1992) Modulation of decay kinetics and frequency of $\mathrm{GABA}_{A}$ receptor-mediated spontaneous inhibitory postsynaptic currents in hippocampal neurons. Neurosci. 49: 1332.

Partridge LD and Swandulla D (1988) Calcium-activated nonspecific cation channels. Trends Neurosci. 11: 69-72.

Perkel DH, Mulloney B and Budelli RW (1981) Quantitative methods for predicting neuronal behavior. Neurosci. 6: 823-827.

Perozo E and Bezanilla F (1990) Phosphorylation affects voltage gating of the delayed rectifier $\mathrm{K}+$ channel by electrostatic interactions. Neuron 5: 685-690.

Press WH, Flannery BP, Teukolsky SA and Vetterling WT (1986) Numerical Recipes. The Art of Scientific Computing. Cambridge University Press, Cambridge, MA.

Rall, W (1967) Distinguishing theoretical synaptic potentials computed for different some-dendritic distributions of synaptic inputs. J. Neurophysiol. 30: 1138-1168.

Raman IM and Trussell LO (1992) The kinetics of the response to glutamate and kainate in neurons of the avian cochlear nucleus. Neuron 9:173-186.

Rinzel J (1985) Excitation dynamics: insights from simplified membrane models. Fed. Proc. 44: 2944-2946.

Rosenmund C, Clements JD and Westbrook GL (1993) Nonuniform probability of release at a hippocampal synapse. Science (Wash.) 262: 754-757.

Ross, EM (1989) Signal sorting and amplification through G protein-coupled receptors. Neuron 3: 141-152.

Sakmann B (1992) Elementary steps in synaptic transmission revealed by currents through single ion channels. Science (Wash.) 256: 503-512.

Sakmann B and Neher E (Editors) (1983) Single-Channel Recording. Plenum Press, New York, NY.

Sansom MSP, Ball FG, Kerry CJ, Ramsey RL and Usherwood PNR (1989) Markov, fractal, diffusion, and related models of ion channel gating. A comparison with experimental data from two ion channels. Biophys. J. 56: 1229-1243.
Sasaki $\mathrm{K}$ and Sato $\mathrm{M}$ (1987) A single GTP-binding protein regulates $\mathrm{K}+$-channels coupled with dopamine, histamine and acetylcholine receptors. Nature 325: 259-262.

Sigworth FJ and Neher E (1980) Single Na channel currents observed in cultured rat muscle cells. Nature 287: 447-449.

Silver RA, Traynelis SF and Cull-Candy SG (1992) Rapid timecourse miniature and evoked excitatory currents at cerebellar synapses in situ. Nature 355: 163-166.

Skene JHP (1990) GAP-43 as a "calmodulin sponge" and some implications for calcium signalling in axon terminals. Neurosci. Res, 13: S112-S125.

Stinivasan R and Chiel HJ (1993) Fast calculation of synaptic conductances. Neural Computation 5, 200-204.

Stevens CF (1978) Interactions between intrinsic membrane protein and electric field. Biophys. J. 22: 295-306.

Standley C, Ramsey RL and Usherwood PNR (1993) Gating kinetics of the quisqualate-sensitive glutamate receptor of locust muscle studied using agonist concentration jumps and computer simulations. Biophys. J. 65: 1379-1386.

Staubli U, Ambros-Ingerson J, Lynch G (1992) Receptor changes and LTP: an analysis using aniracetam, a drug that reversibly modifies glutamate $(A M P A)$ receptors. Hippocampus 2: 49-58.

Steriade M and McCarley RW (1990) Brainstem Control of Wakefulness and Sleep. Plenum Press, New York, NY.

Steriade M, McCormick DA and Sejnowski TJ (1993) Thalamocortical oscillations in the sleeping and aroused brain. Science (Wash.) 262: 679-685.

Strassberg AF and DeFelice LJ (1993) Limitations of the HodgkinHuxley formalism: effects of single channel kinetics on transmembrane voltage dynamics. Neural Computation 5: 843855.

Surprenant A and North RA (1988) Mechanism of synaptic inhibition by noradrenaline acting at alpha 2 -adrenoreceptors. Proc. Roy. Soc. Lond. Ser. B 234: 85-114.

Szabo G and Otero AS (1989) Muscarinic activation of potassium channels in cardiac myocytes: kinetic aspects of $\mathrm{G}$ protein function in vivo. Trends Pharmacol. Sci. Dec. 1989 Suppl.: 46-49.

Thalmann RH (1988) Evidence that guanosine triphosphate (GTP)binding proteins control a synaptic response in brain: effect of pertussis toxin and GTP gamma $S$ on the late inhibitory postsynaptic potential of hippocampal CA 3 neurons. J. Neurosci. 8: $4589-4602$.

Toro L and Stefani E (1991) Calcium-activated K+ channels: metabolic regulation $\mathrm{J}$. Bioenergetics and Biomembranes 23: $561-76$.

Traynelis SF, Silver RA and Cull-Candy SG (1993) Estimated conductance of glutamate receptor channels activated during EPSCs at the cerebellar mossy fiber-granule cell synapse. Neuron 11: 279-289.

Trussell LO and Jackson MB (1987) Dependence of an adenosineactivated potassium current on a GTP-binding protein in mammalian central neurons. J. Neurosci. 7: 3306-3316.

Unwin N (1989) The structure of ion channels in membranes of excitable cells. Neuron 3: 665-676.

Vandenberg CA and Bezanilla $F$ (1991) A model of sodium channel gating based on single channel, macroscopic ionic, and gating currents in the squid giant axon. Biophys. J. 60: 1511-1533.

VanDongen AMJ, Codina J, Olate J, Mattera R, Joho R, Birnbaumer $\mathrm{L}$ and Brown AM (1988) Newly identified brain potas- 
sium channels gated by the guanine nucleotide binding protein $G_{o}$. Science (Wash.) 242: 1433-1437.

Walaas SI and Greengard P (1991) Protein phosphorylation and neuronal function. Pharmacol. Rev. 43: 299-349.

Wang XJ and Rinzel J (1992) Alternating and synchronous rhythms in reciprocally inhibitory model neurons. Neural Computation 4: 84-97.

Wathey JC, Nass MM and Lester HA (1979) Numerical reconstruction of the quantal event at nicotinic synapses. Biophys. J. 27: $145-164$.

Williams JT, Colmers WF and Pan ZZ (1988) Voltage- and ligandactivated inwardly rectifying currents in dorsal raphe neurons in vitro. J. Neurosci. 8: 3499-3506.
Xiang Z, Greenwood AC and Brown T (1992) Measurement and analysis of hippocampal mossy-fiber synapses (abstract). Soc. Neurosci. Abstracts 18: 1350.

Yamada WN, Koch C and Adams PR (1989) Multiple channels and calcium dynamics. In C Koch and I Segev, eds., Methods in Neuronal Modeling. Cambridge, MA: MIT Press, pp. 97-134.

Yamada WM and Zucker RS (1992) Time course of transmitter release calculated from simulations of a calcium diffusion model. Biophys. J. 61: 671-682.

Zador A, Koch C and Brown TH (1990) Biophysical model of a Hebbian synapse. Proc. Natl. Acad. Sci. USA 87: 6718-6722. 